\title{
Binary dislocation junction formation and strength in hexagonal close-packed crystals
}

\author{
Chi-Chin $\mathrm{Wu}^{\mathrm{a},{ }^{*}}$, Sylvie Aubry ${ }^{\mathrm{b}}$, Athanasios Arsenlis ${ }^{\mathrm{b}}$, Peter W. Chung ${ }^{\mathrm{c}, \mathrm{d}}$ \\ ${ }^{a}$ Materials and Manufacturing Science Division, Weapons and Materials Research Directorate, \\ US Army Research Laboratory, Aberdeen Proving Ground, MD 21005, USA \\ ${ }^{b}$ Materials Science Division, Lawrence Livermore National Laboratory P.O. Box 808, L-367 \\ Livermore, CA 94551-0808, USA \\ ${ }^{\mathrm{c}}$ Department of Mechanical Engineering, University of Maryland, College Park, MD 20742, \\ USA \\ ${ }^{\mathrm{d}}$ Computational Sciences Division, Computational and Information Sciences Directorate, US \\ Army Research Laboratory, Adelphi, MD 20783, USA \\ *Corresponding author. Tel: +1 410-306-1481 \\ Email address: chi-chin.wu.ctr@mail.mil (Chi-Chin Wu)
}

\begin{abstract}
This work examines binary dislocation interactions, junction formation and junction strengths in hexagonal close-packed $(h c p)$ crystals. Through a line-tension model and dislocation dynamics (DD) simulations, the interaction and dissociation of different sets of binary junctions are investigated involving one dislocation on the (0110) prismatic plane and a second dislocation on one of the following planes: (0001) basal, (1 $\overline{1} 00)$ prismatic, (1101) primary pyramidal, or $(\overline{2} 112)$ secondary pyramidal. Varying pairs of Burgers vectors are chosen from among the common types: the basal type $\langle a\rangle: \frac{1}{3}\langle 11 \overline{2} 0\rangle$, prismatic type $\langle c\rangle:\langle 0001\rangle$, and pyramidal type $\langle a+c\rangle: \frac{1}{3}\langle 11 \overline{2} \overline{3}\rangle$. For binary interaction due to dislocation intersection, both the analytical results and DD-simulations indicate a relationship between symmetry of interaction maps and the relative magnitude of the Burgers vectors that constitute the junction. Using analytical formulae, a simple regressive model is also developed to represent the junction yield surface. The equation is treated as a degenerated super elliptical equation to quantify the aspect ratio and tilting angle. The results provide analytical insights on binary dislocation interactions that may occur in general $h c p$ metals.
\end{abstract}

\section{Keywords}
A. Dislocations, A. Dynamics, B. Elastic material, C. Analytic functions 


\section{Introduction}

Studies of binary dislocation interactions and junctions largely employed analytical approaches via continuum theories and line tension models. Hirth (1961) originally developed a line tension model to study binary dislocation interactions as a function of slip modes for face-centered cubic $(f c c)$ crystals. The interaction of each possible intersection in the presence of an applied stress was analytically calculated and presented on a Thompson tetrahedron. For those interactions that produced binary junctions, the junction strength, or the stress required to "unzip" the junction, was studied by applying shear stresses on one of the slip planes with the other plane being stress-free. In a study of the overall strain hardening effects of junctions, Schoeck and Frydman (1972), computed the strength of a single junction and used it to estimate the plasticity of a dislocation matrix comprised of identical and equally-separated junctions. More recently, Shanthraj and Zikry (2012) studied the evolution of dislocations due to the increased dislocation density from dislocation interactions including junction formation and annihilations.

With improving computational capabilities, more complex arrangements were later enabled through models (e.g. Pontes et al., 2006) and dislocation dynamics (DD) methods to study strain hardening and dislocation interactions. Devincre and Kubin (1994) considered a network of heterogeneously-distributed junctions in which a junction forms at every intersection where a Frank-Read source cuts across a dislocation forest comprised of identical stationary dislocations. In simulating the reaction events explicitly, they found only about half of the junctions remained after a certain time and their arrangements in the matrix were non-uniform due to the varying local stresses. The reduced population of junctions observed from DD simulations could then be used to modify the line tension 
model to estimate the average junction strength. The same group later demonstrated (Devincre et al., 2008) the quantitative correlations between strain hardening and the individual and collective junction configurations.

Numerous efforts have since examined junctions in different crystals using DD models (e.g. Benzerga et al., 2004; Bertin et al., 2013, 2014; Bulatov et al., 1998, 2006; Capolungo, 2011; Dupuy and Fivel, 2002; Lee et al., 2011; Motz, 2009; Shenoy, 2000; Wickham et al, 1999; Zbib, 2000; Zhou et al., 2011). Other uses of DD simulations indicate that it is a useful means of determining plasticity information using multiscale ideas (e.g. Gao et al., 2011; Liu et al., 2009; Zhou and LeSar, 2012). Among many applications, DD simulations are significantly enriched by including short-range interactions not accounted for in linear elasticity models (Benzerga et al., 2004; Bulatov et al., 1998, 2006; Devincre et al., 2008). Benzerga et al. (2004) developed a two-dimensional model with a special enhancement containing short-range interactions to study binary interactions and resultant hardening. The constitutive equation in the two-dimensional simulation method was modified to yield three-dimensional information. More recently, atomic simulation methods such as molecular dynamics (MD) have been employed. Direct simulations of the zipping and unzipping behaviors (Bulatov, 1998) and unexpected behaviors such as temporary length increase within a junction due to local stress variations (Rodney and Phillips, 1999) have been shown. The blocking effects of junctions proposed by Bulatov and co-workers $(1998,2006)$ via the MD simulations were later verified by Akarapu et al. (2010) who observed in experiments a higher population of tangled dislocations in the immediate vicinity of junctions in a copper $(\mathrm{Cu})$ micropillar. Furthermore, after including the MD-determined core contribution in DD models, Bulatov et al. (2006) obtained a 
junction strength that was comparable to experimental measurements. By combining MD and DD models, Bulatov et al. (2006) also expanded their investigations from binary to multi-junctions and compared the modeled symmetric junction configurations to those observed in molybdenum (Mo) in experiments. Rao et al. (2011) studied binary dislocation intersections using MD simulations alone. In that work, the activation barriers for producing different junctions (the Lomer-Cottrell lock, the Hirth lock and a glissile junction) were evaluated and compared to the experimental values for $\mathrm{Cu}$ by treating the junction configuration as a dislocation cross slip from one plane to the other.

The stability of binary dislocation junctions is sensitive to the slip modes, namely the Burgers vectors, the slip planes of both intersecting dislocations, and the local state of stress. The strength of a junction should therefore be described in terms of these parameters (Bulatov, 1998; Devincre and Kubin, 1994, 2008). Dupuy and Fivel (2002) computed the equilibrium configurations for binary dislocation junctions in $f c c$ crystals based on an improved orientation-dependent line tension model taking into account both the tangential and normal components of the pulling force by a dislocation line. In their model, a force balance adapted from de Wit and Koehler's approach (1959) was also employed to estimate the junction strength. In Dupuy and Fivel's work, the strengths of different junctions in aluminum (Al) were compared via yield surfaces based on shear stress. Despite the absence of dislocation-dislocation interactions, complete yield surface plots were reported for important binary dislocation junctions in $f c c$ crystals.

Junctions formed by dislocation intersections in cubic crystals $(f c c$ and $b c c$ ) were thought to have similar strengths due to the high crystallographic symmetry in $f c c$ and $b c c$ crystals. The noticeable symmetry in the maps of binary interactions (Wickham et al., 
1999) appeared to provide confirmation. Hirth's (1961) calculation of the junction strength as a function of stress on only one plane indicated the dependence of junction strength on the slip mode. Therefore, one may expect more complex junction behavior in $h c p$ crystals given the hexagonal lattice with variable $c / a$ ratios across different materials (where $c$ and $a$ are the vertical and lateral lattice spacings, respectively) and lower crystallographic symmetry than cubic crystals. All of these distinct characters underscore the importance of studying junctions in $h c p$ crystals.

The challenge, therefore, is to systematically investigate a large number of combinations of parameters to establish an inclusive understanding of junctions in $h c p$ crystals. A dislocation generally prefers to slip on the most densely-packed slip plane. This would mean favorable slip on the basal plane for $h c p$ systems (Groh et al., 2009; Wang et al., 2013). However, slip on the prismatic $\{10 \overline{1} 0\}$ and other secondary planes such as typeI $\{10 \overline{1} 1\}$ and type-II $\{11 \overline{2} 2\}$ pyramidal planes, have been both proposed by models (Ando et al., 2002; Chaari et al., 2014) and observed in experiments (Akhtar, 1975; Grinberg et al., 2012). For slip on a type-I pyramidal plane, the Burgers vectors can be either type $\langle a\rangle: \frac{1}{3}\langle 11 \overline{2} 0\rangle$, or type $\langle a+c\rangle: \frac{1}{3}\langle 11 \overline{2} \overline{3}\rangle$ (Yoo et al., 2001). For slip on a type-II plane, only the type $\langle a+c\rangle: \frac{1}{3}\langle 11 \overline{2} \overline{3}\rangle$ Burgers vector having the largest magnitude is available.

A few reports have considered junctions specifically in $h c p$ crystals. Monnet et al (2004) studied binary interactions and examined the effects of dislocation character for zirconium $(\mathrm{Zr})$ via DD simulations. In a manner consistent with theory, the results showed a decrease in the attractive force between the intersecting dislocations when they changed character from edge to screw. The junction map may be used to depict regions in the configuration space, parameterized by the angle each dislocation makes with the line 
created from the intersection of the slip planes, where a junction is most likely to form from a pair of dislocations. The dependence of binary dislocation interactions reported by Monnet et al on the initial dislocation configurations was also similar to what Wickham et al. suggested (1999) for cubic systems. Namely, that junction maps indicate junction formation at the center and four corners regardless of the difference in crystal structures. More recently, Capolungo (2011) studied binary dislocation interactions and junction strengths for varying slip modes in magnesium $(M g)$ via DD simulations using a dislocation mobility adapted from Monnet et al (2004). Capolungo primarily examined the effects of slip including commonly-observed slip planes, with the exception of the type-II pyramidal plane, using the three possible $\langle a\rangle-,\langle c\rangle$ - and $\langle a+c\rangle$-type Burgers vectors. The observations suggested that a general way of understanding junction behavior may exist that is not limited to one specific material but rather is dominated by the distinct crystal structure of $h c p$ metals.

Two earlier papers reported by us studied binary dislocation mechanisms specifically in $M g$ and beryllium (Be) (Wu et al., 2012, 2013). A systematic investigation is performed in the present work on the formation of binary junctions and their strengths in mono-atomic $h c p$ crystals by expanding our investigations to other commonly-occurring $h c p$ materials cadmium $(C d)$, zinc $(Z n)$, cobalt $(C o), Z r$, and titanium $(T i)$. Furthermore, efforts are made to develop an analytical approach to describe interactions and any junction induced by dislocation intersection. A line-tension model has also been integrated into our DD simulation routines. The algorithm estimates an initial configuration that improves calculation efficiency. Furthermore, based on our observations that the yield surfaces are remarkably similar across different materials, a new model relationship is developed to describe junction yield surfaces 
for general mono-atomic hcp crystals. The model is based on a modified form of the orientation-dependent line tension approximation (Dupuy and Fivel, 2002) using a simple factor $\eta$ obtained from isotropic DD simulations. For the sake of convenience, the current effort employs an assumption of isotropy for both the elastic properties and the dislocation mobilities which helps to identify general trends and similarities among different materials and slip parameters.

This paper is organized as follows. Section 2 presents the methodology of this work. The analytical approach based on the line tension model is presented first. A line tension model is used in simplified DD simulations to form binary interaction maps and junction yield surface plots. In Section 3, different approaches are presented to determine analytical binary interaction maps and junction yield surface. A comparison factor $\eta$ is then defined as the ratio of the critical stress obtained by DD simulation with full elasticity to that obtained emulating the line tension approximation to examine the effect of dislocation segmentsegment interactions. The tilting angles and aspect ratios of junction yield surface plots are also computed to numerically verify the similarities of yield surface plots using $B e$ and $M g$ as examples. Finally, a brief conclusion ends the paper in Section 4.

\section{Methodology}

The analytical and numerical methods are described in this section. The equations for interactions between two intersecting straight dislocations are first presented. The description of the method to construct the binary interaction maps then follows. The formulations are then proposed to describe the strength of binary junction in general hcp crystals against destabilization under applied stresses on intersecting slip planes. Following 
analytical descriptions, the method and key parameters used in DD simulation are described for both the formation and destruction of binary junctions. Finally, properties of $h c p$ crystals and the slip systems used in this work for binary junctions are presented.

\subsection{Interaction maps of binary intersecting dislocations}

When two infinitely-long straight dislocations gliding on different slip planes intersect, they may repel, form a junction, or form a stable cross state depending on the interaction force $\boldsymbol{F}_{12}$, as initially derived by Kroupa (1961) and further detailed by Hirth (1982) and depending on glide constraints:

$$
F_{12}\left(\phi_{1_{o}}, \phi_{2_{o}}\right)=\frac{\mu}{\left|T_{1} \times T_{2}\right|} \frac{R_{12}}{\left|R_{12}\right|}\left\{\begin{array}{l}
\frac{1}{2}\left(b_{1} \cdot T_{1}\right)\left(b_{2} \cdot T_{2}\right)-\left(b_{1} \times b_{2}\right) \cdot\left(T_{1} \times T_{2}\right) \\
+\frac{1}{1-v}\left[\left(b_{1} \times T_{1}\right) \cdot \frac{R_{12}}{\left|R_{12}\right|}\right]\left[\left(b_{2} \times T_{2}\right) \cdot \frac{R_{12}}{\left|R_{12}\right|}\right]
\end{array}\right\}
$$

where $\boldsymbol{b}_{1}$ and $\boldsymbol{b}_{2}$ are the Burgers vectors, $\boldsymbol{T}_{1}$ and $\boldsymbol{T}_{2}$ are the dislocation line orientations, and $\phi_{1_{o}}$ and $\phi_{2_{o}}$ are the initial angles each dislocation makes with the junction direction $\boldsymbol{e}_{j}$, defined as $\cos \left(\phi_{1_{o}}\right)=\boldsymbol{T}_{1} \cdot \boldsymbol{e}_{j}$ and $\cos \left(\phi_{2_{o}}\right)=\boldsymbol{T}_{2} \cdot \boldsymbol{e}_{j}$, for the intersecting dislocations denoted as dislocation 1 and dislocation 2. In Eq. (1), $\boldsymbol{R}_{12}=\boldsymbol{T}_{1} \times \boldsymbol{T}_{2}$ is a vector perpendicular to both dislocation line directions, and $\left|\boldsymbol{R}_{12}\right|$ is its magnitude. The junction direction $\boldsymbol{e}_{j}$ can be determined by the two plane normals $\boldsymbol{n}_{1}$ and $\boldsymbol{n}_{2}$ :

$\boldsymbol{e}_{j}=\boldsymbol{n}_{1} \times \boldsymbol{n}_{2}$

where the plane normal $\boldsymbol{n}_{i}(i=1,2)$ is determined from:

$\boldsymbol{n}_{i}=\frac{\boldsymbol{b}_{i} \times \boldsymbol{T}_{i}}{\left|\boldsymbol{b}_{i} \times \boldsymbol{T}_{i}\right|}$

where $\boldsymbol{b}_{\boldsymbol{i}}$ is the Burgers vector and $\boldsymbol{T}_{\boldsymbol{i}}$ is the line direction for dislocation $i$. 
The force $\boldsymbol{F}_{12}$ is written as $F_{12} \boldsymbol{R}_{12}$. The dislocations are repulsive when $F_{12}$ is negative and attractive when $F_{12}$ is positive. When attractive in a zero net stress field, and when it is not kinematically possible to form a junction due to glide constraints, the dislocations may stay in a cross-state where the two dislocations remain in contact at an unbroken common node $\boldsymbol{O}$ (Madec et al., 2002). If the junction formation is favored, node $\boldsymbol{O}$ will split into $\boldsymbol{P}$ and $\boldsymbol{Q}$ along $\boldsymbol{e}_{j}$, and the angles become $\phi_{1}$ and $\phi_{2}$ as depicted in Fig. 1. In the absence of externally-imposed stresses, the two nodes will move along $\boldsymbol{e}_{j}$ until the equilibrium configuration is reached. The illustration also shows the finite lengths of the interacting dislocation arms.

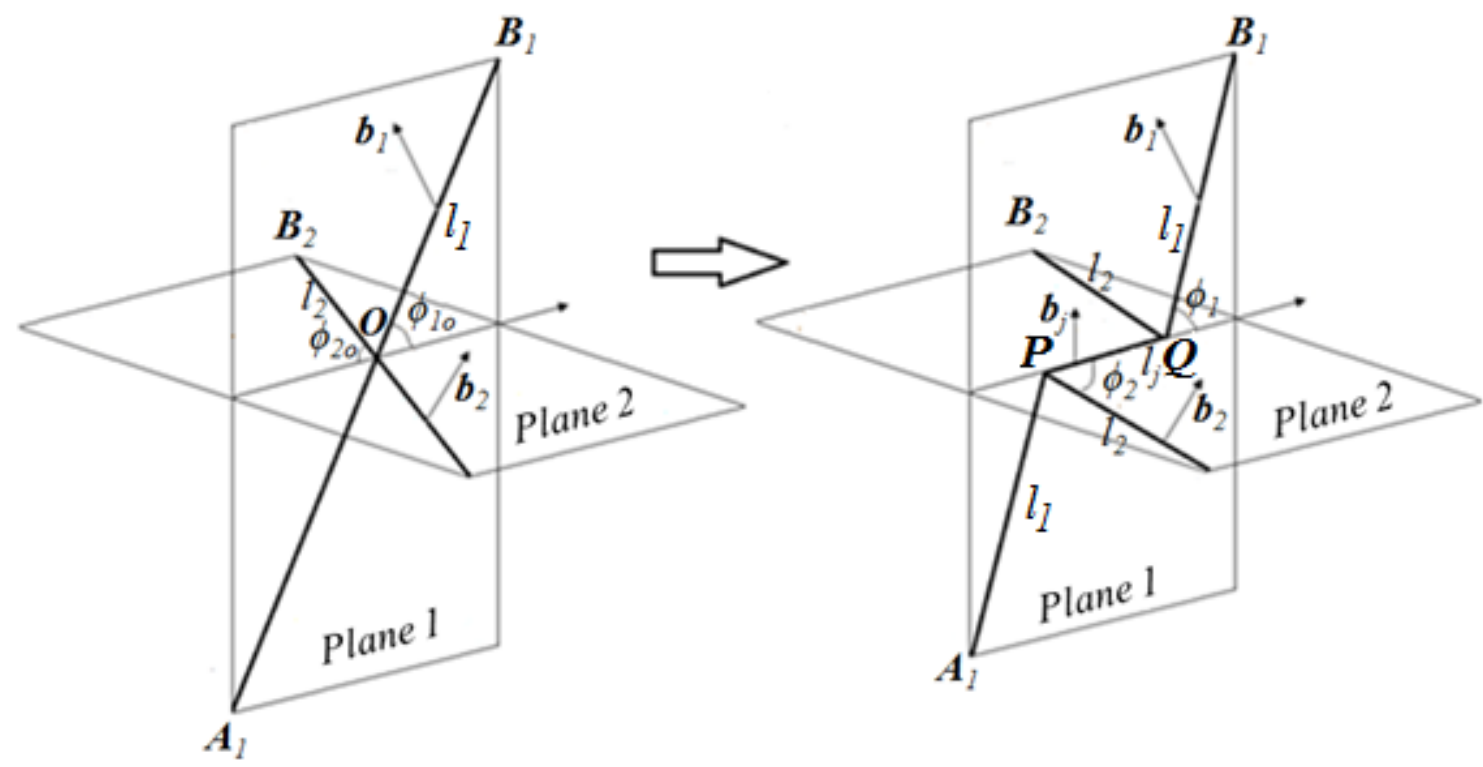

Fig. 1. The formation of a binary junction caused by the intersection of two infinitely long dislocations. $\boldsymbol{A}_{l} \boldsymbol{B}_{1}$ is on plane 1 with a Burgers vector $\boldsymbol{b}_{1}$ and $\boldsymbol{A}_{2} \boldsymbol{B}_{2}$ is on plane 2 with a Burgers vector $\boldsymbol{b}_{2}$.

In this work, we adopt the orientation-dependent line tension model (Dupuy and Fivel, 2002) to determine whether the interactions between the two dislocations lead to junction formation. The dislocation line $i$ is defined by its Burgers vector $\boldsymbol{b}_{i}$ and line 
direction $\boldsymbol{T}_{\boldsymbol{i}}$. Therefore, the screw $\boldsymbol{b}_{i, s}$ and edge $\boldsymbol{b}_{i, e}$ components of Burgers vectors can be defined as:

$\boldsymbol{b}_{i, s}=\left(\boldsymbol{b}_{i} \cdot \boldsymbol{T}_{i}\right) \boldsymbol{T}_{i}=b_{i, s} \boldsymbol{T}_{i}$

$\boldsymbol{b}_{i, e}=b_{i, e} \boldsymbol{M}_{i}=\boldsymbol{b}_{i}-b_{i, s} \boldsymbol{T}_{i}$

Here, the $\boldsymbol{M}_{\boldsymbol{i}}$ is the unit vector normal to the dislocation line direction $\boldsymbol{T}_{\boldsymbol{i}}$ on the glide plane. The magnitude of the edge component can then be calculated from $b_{i, e}^{2}=b_{i}^{2}-b_{i, s}^{2}$. If $\mu$ is the shear modulus, $v$ is the Poisson ratio, and $R$ and $r_{o}$ are the outer and inner cutoff radii, the line tension energy can be used to approximate the elastic energy per unit length for a straight dislocation $i$ given by (Hirth and Lothe, 1982):

$E\left(\boldsymbol{b}_{i}, \boldsymbol{T}_{i}\right)=\frac{\mu}{4 \pi(1-v)} \ln \left(\frac{R}{r_{o}}\right)\left[b_{i, e}^{2}+b_{i, s}^{2}(1-v)\right]=K\left[b_{i, e}^{2}+b_{i, s}^{2}(1-v)\right]$

The parameter $K$ in Eq. (6) is defined as:

$$
K=\frac{\mu}{4 \pi(1-v)} \ln \left(\frac{R}{r_{o}}\right)
$$

Therefore, any point of the dislocation $i$ experiences a pulling force due to line tension, denoted by $\boldsymbol{F}_{i}$. The line tension force is composed of the sum of a force component $\boldsymbol{F}_{i, T}$ in the tangent direction $\boldsymbol{T}_{i}$, and a force component $\boldsymbol{F}_{i, M}$ in the normal direction $\boldsymbol{M}_{i}$ (Dupuy and Fivel, 2002) given as:

$\boldsymbol{F}_{i}=\boldsymbol{F}_{i, T}+\boldsymbol{F}_{i, M}=E \boldsymbol{T}_{i}+E^{\prime} \boldsymbol{M}_{i}$

where

$$
\begin{aligned}
& \boldsymbol{F}_{i, T}=E \boldsymbol{T}_{i}=K\left[b_{i, s}^{2}(1-v)+b_{i, e}^{2}\right] \boldsymbol{T}_{i} \\
& \boldsymbol{F}_{i, M}=E^{\prime} \boldsymbol{M}_{i}=2 v K b_{i, s} b_{i, e} \boldsymbol{M}_{i}
\end{aligned}
$$

When it is energetically favorable for the junction to form, node $\boldsymbol{O}$ will split into two triple nodes, $\boldsymbol{P}$ and $\boldsymbol{Q}$ (as seen in Fig. 1), and travel along the line where the two glide planes 
intersect in the directions of $\boldsymbol{e}_{j}$ and $-\boldsymbol{e}_{j}$. The net force magnitude at either junction triple node can be determined to be:

$$
F_{e q}\left(\boldsymbol{T}_{i}, l_{i}\right)=\boldsymbol{F}_{j}\left(-\boldsymbol{e}_{j}, \boldsymbol{b}_{j}\right) \cdot \boldsymbol{e}_{j}+\sum_{i=1,2} \boldsymbol{F}_{i}\left(\boldsymbol{T}_{i}, \boldsymbol{b}_{i}\right) \cdot \boldsymbol{e}_{j}
$$

where $\boldsymbol{F}_{j}$ is the line tension of junction having a Burgers vector $\boldsymbol{b}_{j}\left(\boldsymbol{b}_{j}=\boldsymbol{b}_{1}+\boldsymbol{b}_{2}\right)$ and $\boldsymbol{F}_{i}$ is the line tension for each dislocation arm. Eq. (11) is a force balance at the triple node between the force exerted by the junction and forces from the two dislocation arms. When $F_{e q}$ is positive, the junction will grow until $F_{e q}$ vanishes or when all forces are balanced at the triple node. Since the dislocation line directions, $\boldsymbol{T}_{1}$ and $\boldsymbol{T}_{2}$ are determined by the initial angle each dislocation makes with $\boldsymbol{e}_{j}, \phi_{1 o}$ and $\phi_{2 o}$, the binary interaction due to dislocation intersection can be depicted via two-dimensional maps through Eq. (11) using the variable $\phi_{i o}(i=1,2)$ and a discrete-valued function $G\left(\phi_{10}, \phi_{20}\right)$ :

1. $G\left(\phi_{1 o}, \phi_{2 o}\right)=1$ : A junction will form if $F_{e q}>0$.

2. $G\left(\phi_{1 o}, \phi_{2 o}\right)=0$ : No junction will form. Instead, the dislocations form a cross-state if

$$
F_{12}\left(\phi_{1 o}, \phi_{2 o}\right) \geq 0 \text { and } F_{e q} \leq 0 \text {. }
$$

3. $G\left(\phi_{1 o}, \phi_{2 o}\right)=-1$ : Neither a junction nor a cross-state will form if $F_{12}\left(\phi_{1_{o}}, \phi_{2_{o}}\right)<0$ and $F_{e q} \leq 0$

A sufficient condition for a junction to form is the sign on $F_{e q}$, namely $F_{e q}>0$. It is furthermore noteworthy that the formation is independent of the sign of $F_{12}\left(\phi_{1 o}, \phi_{2 o}\right)$. Thus, repulsive interaction between two intersecting parent dislocations may still result in the formation of a stable junction.

Plots of the function $G$ constitute analytical junction maps for the different slip systems. For each angle pair $\phi_{1 o}$ and $\phi_{2 o}$, the value of G can be depicted using three types of symbols to represent the three types of formation. Local regions of one type of symbols can reasonably 
approximate a continuous region in the map for that formation. Increasing the number of angle pairs will make the junction map interior boundaries smoother.

\subsection{The junction yield surfaces}

Starting with Eq. (11) for $F_{e q}=0$, the objective is to portray the junction yield surface as a function of the shear stresses $\sigma_{1}$ and $\sigma_{2}$, the material parameters, Burgers vectors, and the equilibrium junction configuration. It will be shown that the yield surface may be approximated by a third order polynomial expression in terms of stress. However, the shape of the yield surface resembles a degenerated ellipse with more pointy ends along the major axis in appearance but having identical tilting angle and aspect ratio. We therefore relate this ellipse to the yield surface and compute the tilting angle and the major and minor axes by using the parameters of ellipse. As a result, we arrive at a simple heuristic model containing a small number of parameters. Here, we describe the relevant formulation of the line tension model followed by comparing with the general equation of an ellipse to approximate the line tension result.

\subsubsection{Line tension model for the yield surface}

Given that the junction yield surface is determined as a function of $\sigma_{1}$ and $\sigma_{2}$, the present effort is limited to stress states that do not result in a net stress on the junction. The junction segment remains straight under the influence of the applied stress field in most cases unless the dislocation interactions generate local stresses on the glide plane of the junction during the "unzipping" process (Wu et al., 2013). Thus, the forces experienced by the portion

of junction line at the intersection edge depend only on the Burgers vector $\boldsymbol{b}_{j}$ since the line 
direction of the junction between the two triple nodes does not change prior to and during unzipping. Thus, the forces simplify as follows:

$\boldsymbol{F}_{j, M}=0$

$\boldsymbol{F}_{j}=\boldsymbol{F}_{j, T}=E\left(\boldsymbol{b}_{j}, \boldsymbol{T}_{j}\right) \boldsymbol{T}_{j}=E\left(\boldsymbol{b}_{j}, \boldsymbol{e}_{j}\right) \boldsymbol{e}_{j}$

The stresses needed to unzip a junction that is in equilibrium are those that bring Eq. (11) out of balance. Once the triple node becomes unstable due to the imbalanced forces, the junction begins to unzip - an action where the triple nodes move along the junction line either toward one another or along the same direction depending on the applied stress field. Figure 2 schematically illustrates the junction configuration with arms that have bowed on their respective planes due to an applied stress field. The direction of bow-out of each arm depends on the resolved stress on its glide plane. The configuration shown in Fig. 2 assumes a net stress field that produces resolved stresses in the direction favoring junction unzipping. The bowing dislocation arms subsequently create angles $\alpha_{1}$ and $\alpha_{2}$ to their initial line directions at the triple node.

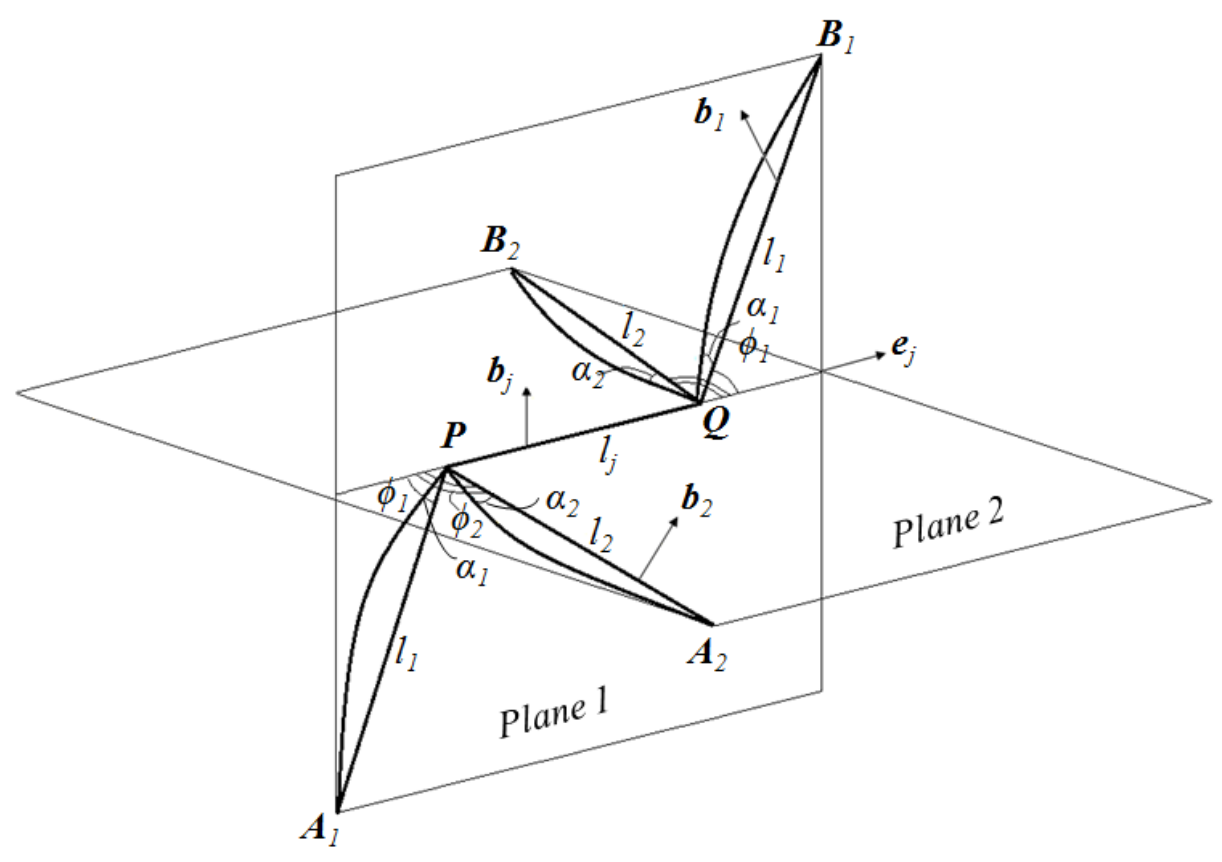


Fig. 2. A diagram showing the configuration of all arms at junction triple nodes under an applied stress field. $\alpha_{1}$ and $\alpha_{2}$ are the bow out angles with respect to the originally straight lines for dislocations $1\left(l_{1}\right)$ and $2\left(l_{2}\right)$, respectively. $\phi_{1}$ and $\phi_{2}$ are the angles that the tangent directions of bowed dislocation arms make to $\boldsymbol{e}_{j}$ for dislocations 1 and 2 , respectively. $l_{j}$ is the junction, $\boldsymbol{b}_{1}, \boldsymbol{b}_{2}$, and $\boldsymbol{b}_{j}$ are the Burgers vectors for $l_{1}$ and $l_{2}$, and $l_{j}$, respectively.

Under a general applied stress field $\sigma_{i}$, the Peach-Koehler $(P-K)$ force per unit length exerted on a dislocation $\operatorname{arm} i$ is:

$\boldsymbol{F}_{i}^{P K}=\left(\boldsymbol{\sigma}_{i} \cdot \boldsymbol{b}_{i}\right) \times \boldsymbol{T}_{i}^{\prime}$

where the $\boldsymbol{T}_{i}^{\prime}$ is the new tangent direction of dislocation arm at the triple node due to the bowout. During "unzipping", the triple node moves along the junction line which involves only the glide components of $P-K$ forces (Hirth and Lothe, 1982). The minimum applied stresses $\left(\sigma_{1}, \sigma_{2}\right)$ required to destabilize the equilibrium junction must therefore satisfy the following force balance at the triple node:

$0=\boldsymbol{F}_{j, T}\left(\boldsymbol{b}_{j}\right) \cdot \boldsymbol{e}_{j}+\sum_{i=1,2}\left[\boldsymbol{F}_{i, T}\left(\boldsymbol{T}_{i}^{\prime}, \boldsymbol{b}_{i}\right)+\boldsymbol{F}_{i, M}\left(\boldsymbol{T}_{i}^{\prime}, \boldsymbol{b}_{i}\right)-\boldsymbol{F}_{i, g}^{P K}\right] \cdot \boldsymbol{e}_{j}$

Substituting Eqs. (9), (10) and (14) into Eq. (15) yields:

$0=E\left(\boldsymbol{b}_{j}, \boldsymbol{e}_{j}\right) \boldsymbol{T}_{j} \cdot \boldsymbol{e}_{j}+\sum_{i=1,2}\left\{E\left(\boldsymbol{b}_{i}, \boldsymbol{T}_{i}^{\prime}\right) \boldsymbol{T}_{i}^{\prime} \cdot \boldsymbol{e}_{j}+\left(E^{\prime}\left(\boldsymbol{b}_{i}, \boldsymbol{T}_{i}^{\prime}\right)-F_{i, g}^{P K}\right) \boldsymbol{M}_{i}^{\prime} \cdot \boldsymbol{e}_{j}\right\}$

Eq. (16) defines a locus of points for variables $\sigma_{1}$ and $\sigma_{2}$ (i.e., the junction yield surface) that satisfy the equality as a function of the slip system and dislocation orientations associated with the junction. Here, the tangent and normal directions to the dislocation arm, $\boldsymbol{T}_{i}^{\prime}$ and $\boldsymbol{M}_{i}^{\prime}$ may have changed from $\boldsymbol{T}_{i}$ and $\boldsymbol{M}_{i}$ due to bow-out angle $\alpha_{i}$ depending on the resolved applied stress $\sigma_{i}$. Assuming $\left|d \alpha_{i}\right| \ll 1$, the bow-out curvature, $R_{i}$, of each arm can be calculated:

$R_{i}=\frac{E\left(\boldsymbol{b}_{i}, \boldsymbol{T}_{i}\right)}{\sigma_{i} b_{i}}=\frac{K\left[b_{i, e}^{2}+b_{i, s}^{2}(1-v)\right]}{\sigma_{i} b_{i}}$ 
where $E$ is the line tension energy per unit length as shown in Eq. (6). Then, the bow-out angle $\alpha_{i}$ can be calculated from $R_{i}$ as:

$\alpha_{i}=\sin ^{-1}\left(\frac{l_{i}}{2 R_{i}}\right)$

Correlating $\boldsymbol{T}_{i}^{\prime}$ and $\boldsymbol{M}_{i}^{\prime}$ to $\boldsymbol{T}_{i}$ and $\boldsymbol{M}_{i}$ through the bow-out angle $\alpha_{i}$, and calculating $E\left(\boldsymbol{b}_{i}, \boldsymbol{T}_{i}^{\prime}\right)$ and $E^{\prime}\left(\boldsymbol{b}_{i}, \boldsymbol{T}_{i}^{\prime}\right)$ from Eqs. (9) and (10), a rather cumbersome expression results that can be seen as a polynomial assuming small bow out angles (Hull and Bacon, 1984). After some simplifications, Eq. (16) finally becomes:

$$
\sum_{i=1,2}\left\{U_{i} \tau_{i}^{3}+V_{i} \tau_{i}^{2}+W_{i} \tau_{i}+\left(X_{i} \tau_{i}^{2}+Y_{i} \tau_{i}+Z_{i}\right) \sqrt{1-\tau_{i}^{2}}\right\}-E\left(\boldsymbol{b}_{j}, \boldsymbol{e}_{j}\right)=0
$$

where

$$
\begin{gathered}
U_{i}=3 v K\left[2 b_{i, s} b_{i, e} \boldsymbol{T}_{i} \cdot \boldsymbol{e}_{j}+\left(b_{i, e}^{2}-b_{i, s}^{2}\right)\left|\boldsymbol{T}_{i} \times \boldsymbol{e}_{j}\right|\right] \\
=3 E^{\prime}\left(\boldsymbol{b}_{i}, \boldsymbol{T}_{i}\right) \boldsymbol{T}_{i} \cdot \boldsymbol{e}_{j}+3 v K\left(b_{i, e}^{2}-b_{i, s}^{2}\right)\left|\boldsymbol{T}_{i} \times \boldsymbol{e}_{j}\right| \\
V_{i}=-\frac{E\left(\boldsymbol{b}_{i}, \boldsymbol{T}_{i}\right)}{l_{i}}\left(\boldsymbol{T}_{i} \cdot \boldsymbol{e}_{j}\right)\left|\boldsymbol{T}_{i} \times \boldsymbol{e}_{j}\right| \\
W_{i}=K\left\{-4 v b_{i, s} b_{i, e} \boldsymbol{T}_{i} \cdot \boldsymbol{e}_{j}+\left[(3 v-1) b_{i, e}^{2}-(2 v+1) b_{i, s}^{2}\right]\left|\boldsymbol{T}_{i} \times \boldsymbol{e}_{j}\right|\right\} \\
=-2 E^{\prime}\left(\boldsymbol{b}_{i}, \boldsymbol{T}_{i}\right) \boldsymbol{T}_{i} \cdot \boldsymbol{e}_{j}+\left[3 v K\left(b_{i, e}^{2}-b_{i, s}^{2}\right)-E\right]\left|\boldsymbol{T}_{i} \times \boldsymbol{e}_{j}\right| \\
X_{i}= \pm 3 v K\left[\left(b_{i, s}^{2}-b_{i, e}^{2}\right) \boldsymbol{T}_{i} \cdot \boldsymbol{e}_{j}+2 b_{i, s} b_{i, e}\left|\boldsymbol{T}_{i} \times \boldsymbol{e}_{j}\right|\right] \\
= \pm 3 v K\left(b_{i, s}^{2}-b_{i, e}^{2}\right) \boldsymbol{T}_{i} \cdot \boldsymbol{e}_{j} \pm 3 E^{\prime}\left(\boldsymbol{b}_{i}, \boldsymbol{T}_{i}\right)\left|\boldsymbol{T}_{i} \times \boldsymbol{e}_{j}\right| \\
Y_{i}=\frac{\mp E\left(\boldsymbol{b}_{i}, \boldsymbol{T}_{i}\right)}{l_{i}}\left|\boldsymbol{T}_{i} \times \boldsymbol{e}_{j}\right|^{2} \\
Z_{i}= \pm K\left\{-2 v b_{i, s} b_{i, e}\left|\boldsymbol{T}_{i} \times \boldsymbol{e}_{j}\right|+\left(b_{i, e}^{2}+(1-v) b_{i, s}^{2}\right) \boldsymbol{T}_{i} \cdot \boldsymbol{e}_{j}\right\} \\
=\mp E^{\prime}\left(\boldsymbol{b}_{i}, \boldsymbol{T}_{i}\right)\left|\boldsymbol{T}_{i} \times \boldsymbol{e}_{j}\right| \pm E\left(\boldsymbol{b}_{i}, \boldsymbol{T}_{i}\right) \boldsymbol{T}_{i} \cdot \boldsymbol{e}_{j}
\end{gathered}
$$


and $E\left(\boldsymbol{b}_{i}, \boldsymbol{T}_{i}\right)$ and $E\left(\boldsymbol{b}_{j}, \boldsymbol{e}_{j}\right)$ are as defined in Eq. (6) for the arms and the junction segments, respectively. In Eq. (19), the $X_{i}$ and $Z_{i}$ coefficients take the positive sign and the $Y_{i}$ coefficient takes the negative sign when $0 \leq \alpha_{i}<\frac{\pi}{2}$. On the other hand, the $X_{i}$ and $Z_{i}$ coefficients take the negative sign and the $Y_{i}$ coefficient takes the positive sign when $-\frac{\pi}{2} \leq \alpha_{i}<0$. The variable, $\tau_{i}$, is the normalized resolved shear stress that accounts for the initial length $l_{i}$ of the dislocation arm and its line tension:

$\tau_{i}=\frac{l_{i} b_{i}}{2 K\left(b_{i, e}^{2}+(1-v) b_{i, s}^{2}\right)} \sigma_{i}=\frac{l_{i} b_{i}}{2 E\left(\boldsymbol{b}_{i,} \boldsymbol{T}_{i}\right)} \sigma_{i}$

The equilibrium arm lengths $l_{i}$ can be determined from its initial length $l_{i o}$ :

$l_{i}=l_{i o} \frac{\left|\boldsymbol{T}_{i o} \times \boldsymbol{e}_{j}\right|}{\left|\boldsymbol{T}_{i} \times \boldsymbol{e}_{j}\right|}$

where $\boldsymbol{T}_{i o}$ is the initial line direction of dislocation $i$ while intersecting each other. Eq. (19) is effectively a polynomial of order three in $\tau_{i}$. The closed and continuous envelope drawn for $\sigma_{l}$ and $\sigma_{2}$ that satisfies the equality represents the junction yield surface.

\subsubsection{Elliptical approximation of the yield surface}

The surface has visibly similar features to a superellipse, namely a Lamé curve, given by:

$\left|\frac{\tau_{1}}{p}\right|^{\lambda}+\left|\frac{\tau_{2}}{q}\right|^{\lambda}=1$

where $\lambda$ is a real number. Based on this observation, a heuristic relationship can be developed by approximating Eq. (19) with an egg curve evolved from a second order ellipse equation with $\tau_{1}$ and $\tau_{2}$ as variables that rotate $\beta$ (in degrees) clockwise from the $x y$-coordinate system (Kalman, 2008): 
$\frac{\left(\tau_{1} \cos \beta+\tau_{2} \sin \beta\right)^{2}}{p^{2}}+\frac{\left(\tau_{1} \sin \beta-\tau_{2} \cos \beta\right)^{2}}{q^{2}}=0$

Here, $\beta$ corresponds to the tilting angle and $p$ and $q$ are the major and minor axes for the yield surface. After rearranging the terms, Eq. (23) becomes:

$\left(\frac{\cos ^{2} \beta}{p^{2}}+\frac{\sin ^{2} \beta}{q^{2}}\right) \tau_{1}^{2}-\sin 2 \beta\left(\frac{1}{p^{2}}-\frac{1}{q^{2}}\right) \tau_{1} \tau_{2}+\left(\frac{\sin ^{2} \beta}{p^{2}}+\frac{\cos ^{2} \beta}{q^{2}}\right) \tau_{2}^{2}-1=0$

As a result, the three values $p, q$, and $\beta$ serve to parameterize a simple function that approximates the actual yield surface and can be determined through a rough fit of Eq. (24) to Eq. (19). Since we treat Eq. (19) as a degenerated third order super-ellipse expression, the third order terms in Eq. (19) can then be used to correlate to the $\tau_{1}^{2}$ and $\tau_{2}^{2}$ terms in Eq. (24) after dividing by $E\left(\boldsymbol{b}_{j}, \boldsymbol{e}_{j}\right)$. Therefore, the sum of coefficients $U_{i}$ and $X_{i}$ in Eq. (19) correspond to the coefficients $\left(\frac{\cos ^{2} \beta}{p^{2}}+\frac{\sin ^{2} \beta}{q^{2}}\right)$ and $\left(\frac{\sin ^{2} \beta}{p^{2}}+\frac{\cos ^{2} \beta}{q^{2}}\right)$ in Eq. (24) for dislocations 1 and 2 , respectively, in the following way:

$$
\left(\frac{\cos ^{2} \beta}{p^{2}}+\frac{\sin ^{2} \beta}{q^{2}}\right)=\frac{3 v K}{E\left(\boldsymbol{b}_{j}, \boldsymbol{e}_{j}\right)}\left\{\begin{array}{c}
\left(2 b_{1, s} b_{1, e} \pm b_{1, s}^{2} \mp b_{1, e}^{2}\right)\left(\boldsymbol{T}_{1} \cdot \boldsymbol{e}_{j}\right) \\
+\left(b_{1, e}^{2}-b_{1, s}^{2} \pm 2 b_{1, s} b_{1, e}\right)\left|\boldsymbol{T}_{1} \times \boldsymbol{e}_{j}\right|
\end{array}\right\}
$$

and

$$
\left(\frac{\sin ^{2} \beta}{p^{2}}+\frac{\cos ^{2} \beta}{q^{2}}\right)=\frac{3 v K}{E\left(\boldsymbol{b}_{j}, \boldsymbol{e}_{j}\right)}\left\{\begin{array}{c}
\left(2 b_{2, s} b_{2, e} \pm b_{2, s}^{2} \mp b_{2, e}^{2}\right)\left(\boldsymbol{T}_{2} \cdot \boldsymbol{e}_{j}\right) \\
+\left(b_{2, e}^{2}-b_{2, s}^{2} \pm 2 b_{2, s} b_{2, e}\right)\left|\boldsymbol{T}_{2} \times \boldsymbol{e}_{j}\right|
\end{array}\right\}
$$

Adding and subtracting Eqs. (25) and (26) leads to

$$
\begin{gathered}
\frac{1}{p^{2}}+\frac{1}{q^{2}}=\frac{3 v K}{E\left(\boldsymbol{b}_{j}, \boldsymbol{e}_{j}\right)}\left\{\left(2 b_{1, s} b_{1, e} \pm b_{1, s}^{2} \mp b_{1, e}^{2}\right)\left(\boldsymbol{T}_{1} \cdot \boldsymbol{e}_{j}\right)+\left(b_{1, e}^{2}-b_{1, s}^{2} \pm 2 b_{1, s} b_{1, e}\right)\left|\boldsymbol{T}_{1} \times \boldsymbol{e}_{j}\right|\right. \\
\left.+\left(2 b_{2, s} b_{2, e} \pm b_{2, s}^{2} \mp b_{2, e}^{2}\right)\left(\boldsymbol{T}_{2} \cdot \boldsymbol{e}_{j}\right)+\left(b_{2, e}^{2}-b_{2, s}^{2} \pm 2 b_{2, s} b_{2, e}\right)\left|\boldsymbol{T}_{2} \times \boldsymbol{e}_{j}\right|\right\}
\end{gathered}
$$

and 


$$
\frac{1}{p^{2}}-\frac{1}{q^{2}}=\frac{1}{\cos (2 \beta)}\left(\frac{1}{p^{2}}+\frac{1}{q^{2}}\right)
$$

Therefore, the coefficient for the $\tau_{1} \tau_{2}$ term in Eq. (24) can be rewritten using Eqs. (27) and (28) such that:

$$
\begin{aligned}
& -\sin 2 \beta\left(\frac{1}{p^{2}}-\frac{1}{q^{2}}\right)= \\
& \frac{-3 v K \tan (2 \beta)}{E\left(\boldsymbol{b}_{j}, \boldsymbol{e}_{j}\right)}\left\{\begin{array}{c}
\left(2 b_{1, s} b_{1, e} \pm b_{1, s}^{2} \mp b_{1, e}^{2}\right)\left(\boldsymbol{T}_{1} \cdot \boldsymbol{e}_{j}\right)-\left(2 b_{2, s} b_{2, e} \pm b_{2, s}^{2} \mp b_{2, e}^{2}\right)\left(\boldsymbol{T}_{2} \cdot \boldsymbol{e}_{j}\right) \\
+\left(b_{1, e}^{2}-b_{1, s}^{2} \pm 2 b_{1, s} b_{1, e}\right)\left|\boldsymbol{T}_{1} \times \boldsymbol{e}_{j}\right|-\left(b_{2, e}^{2}-b_{2, s}^{2} \pm 2 b_{2, s} b_{2, e}\right)\left|\boldsymbol{T}_{2} \times \boldsymbol{e}_{j}\right|
\end{array}\right\}
\end{aligned}
$$

The $\tau_{1} \tau_{2}$ in Eq. (24) and lower order terms in Eq. (19) relate to the tilt of the ellipse. Therefore, the right side in Eq. (29) corresponds to the sum of all coefficients lower than third order in Eq. (24), namely, adding $V_{i}, W_{i}, Y_{i}$ and $Z_{i}$ results in:

$$
\begin{aligned}
& \sum_{i=1,2}\left\{-\frac{E\left(\boldsymbol{b}_{i}, \boldsymbol{T}_{i}\right)}{l_{i}}\left(\boldsymbol{T}_{i} \cdot \boldsymbol{e}_{j}\right)\left|\boldsymbol{T}_{i} \times \boldsymbol{e}_{j}\right|-4 v K b_{i, s} b_{i, e}\left(\boldsymbol{T}_{i} \cdot \boldsymbol{e}_{j}\right)+\left[(3 v-1) K b_{i, e}^{2}-(2 v+\right.\right. \\
& \begin{array}{c}
\left.1) K b_{i, s}^{2}\right]\left|\boldsymbol{T}_{i} \times \boldsymbol{e}_{j}\right| \mp \frac{E\left(\boldsymbol{b}_{i}, \boldsymbol{T}_{i}\right)}{l_{i}}\left|\boldsymbol{T}_{i} \times \boldsymbol{e}_{j}\right|^{2} \mp 2 v K b_{i, s} b_{i, e}\left|\boldsymbol{T}_{i} \times \boldsymbol{e}_{j}\right| \pm K\left(b_{i, e}^{2}+(1-v) b_{i, s}^{2}\right)\left(\boldsymbol{T}_{i} \cdot\right. \\
\left.\left.\boldsymbol{e}_{j}\right)\right\}=\sum_{i=1,2}\left\{-\frac{E\left(\boldsymbol{b}_{i}, \boldsymbol{T}_{i}\right)}{l_{i}}\left(\boldsymbol{T}_{i} \cdot \boldsymbol{e}_{j}\right)\left|\boldsymbol{T}_{i} \times \boldsymbol{e}_{j}\right|+K\left[ \pm b_{i, e}^{2}+(1-v) b_{i, s}^{2}-4 v b_{i, s} b_{i, e}\right]\left(\boldsymbol{T}_{i} \cdot \boldsymbol{e}_{j}\right)+\right. \\
\left.K\left[(3 v-1) b_{i, e}^{2}-(2 v+1) b_{i, s}^{2} \mp 2 v b_{i, s} b_{i, e}\right]\left|\boldsymbol{T}_{i} \times \boldsymbol{e}_{j}\right| \mp \frac{E\left(\boldsymbol{b}_{i}, \boldsymbol{T}_{i}\right)}{l_{i}}\left|\boldsymbol{T}_{i} \times \boldsymbol{e}_{j}\right|^{2}\right\}= \\
\sum_{i=1,2}\left\{\begin{array}{c}
-\frac{E\left(\boldsymbol{b}_{i}, \boldsymbol{T}_{i}\right)}{l_{i}}\left[\left(\boldsymbol{T}_{i} \cdot \boldsymbol{e}_{j}\right)\left|\boldsymbol{T}_{i} \times \boldsymbol{e}_{j}\right| \pm\left|\boldsymbol{T}_{i} \times \boldsymbol{e}_{j}\right|^{2}\right]+ \\
K\left[ \pm(1-v) b_{i, s}^{2}-4 v b_{i, s} b_{i, e} \pm b_{i, e}^{2}\right]\left(\boldsymbol{T}_{i} \cdot \boldsymbol{e}_{j}\right) \\
+K\left[(3 v-1) b_{i, e}^{2} \mp 2 v b_{i, s} b_{i, e}-(2 v+1) b_{i, s}^{2}\right]\left|\boldsymbol{T}_{i} \times \boldsymbol{e}_{j}\right|
\end{array}\right\}
\end{array}
\end{aligned}
$$

Equating Eq. (29) and Eq. (30) gives: 


$$
\left.-\tan 2 \beta=\frac{\sum_{i=1,2}\left\{\begin{array}{c}
-\frac{E\left(\boldsymbol{b}_{i}, \boldsymbol{T}_{i}\right)}{l_{i}}\left[\left(\boldsymbol{T}_{i} \cdot \boldsymbol{e}_{j}\right)\left|\boldsymbol{T}_{i} \times \boldsymbol{e}_{j}\right| \pm\left|\boldsymbol{T}_{i} \times \boldsymbol{e}_{j}\right|^{2}\right]+ \\
K\left[ \pm(1-v) b_{i, s}^{2}-4 v b_{i, s} b_{i, e} \pm b_{i, e}^{2}\right]\left(\boldsymbol{T}_{i} \cdot \boldsymbol{e}_{j}\right) \\
+K\left[(3 v-1) b_{i, e}^{2} \mp 2 v b_{i, s} b_{i, e}-(2 v+1) b_{i, s}^{2}\right]\left|\boldsymbol{T}_{i} \times \boldsymbol{e}_{j}\right|
\end{array}\right\} \frac{E\left(\boldsymbol{b}_{j}, \boldsymbol{e}_{j}\right)}{3 v K}}{\left\{\begin{array}{l}
\left(2 b_{1, s} b_{1, e} \pm b_{1, s}^{2} \mp b_{1, e}^{2}\right)\left(\boldsymbol{T}_{1} \cdot \boldsymbol{e}_{j}\right) \\
+\left(b_{1, e}^{2}-b_{1, s}^{2} \pm 2 b_{1, s} b_{1, e}\right)\left|\boldsymbol{T}_{1} \times \boldsymbol{e}_{j}\right| \\
-\left(2 b_{2, s} b_{2, e} \pm b_{2, s}^{2} \mp b_{2, e}^{2}\right)\left(\boldsymbol{T}_{2} \cdot \boldsymbol{e}_{j}\right) \\
+\left(b_{2, e}^{2}-b_{2, s}^{2} \pm 2 b_{2, s} b_{2, e}\right)\left|\boldsymbol{T}_{2} \times \boldsymbol{e}_{j}\right|
\end{array}\right.}\right\}
$$

Eq. (31) provides the relation for calculating the titling angle $\beta$. When the denominator vanishes, the titling angle $\beta=\pi / 4,3 \pi / 4, \ldots$, etc.

After obtaining the tilting angle $\beta$, the major and minor axes $p$ and $q$ for a yield surface can then be calculated using Eq. (27) and Eq. (28).

\subsection{Discrete Dislocation Simulations for binary interactions}

The hcp crystal structure was recently added to the DD simulator, ParaDiS (Arsenlis et al., 2007, Aubry et al) based on Damanio's description of Burgers vectors and planes in the hep structure (Damiano, 1963). In the DD method, dislocation lines are discretized into straight segments joined by nodes. Some nodes are called discretization nodes; they are created to represent continuous dislocation lines. These nodes are connected to two other nodes by segments of the same Burgers vector. Multi-arm nodes, called $n$-nodes or multinodes are physical nodes. They connect three or more segments with more than two distinct Burgers vectors.

\subsubsection{Binary junction interaction}


To investigate junction formation, two straight dislocations with pinned endpoints and of same length (40,000a, where $a$ is the lattice spacing in the lateral direction) are placed on different slip planes for each pair of slip systems examined. The dislocations are initially placed near each other at their midpoints with 30-degree intersecting angles to the junction direction $\boldsymbol{e}_{j}$, namely $\phi_{1 o}=\phi_{2 o}=30^{\circ}$, having a small separation distance with each other along the direction of $\boldsymbol{T}_{1} \times \boldsymbol{T}_{2}$. (Several separation distances were used ranging from 10a to 100a, no difference in the behavior of the interaction forces were observed as a function of the separation distance.) If the interaction is attractive, the two dislocations will meet and form a 4-node that will either remain in the 4-node cross-state or, when it is energetically favorable, break into two 3-nodes connected by a junction. When the interaction is repulsive, two scenarios may happen. The two interacting dislocations may move apart on their respective glide planes so that no junction would form or a junction would form if it is energetically favorable.

\subsubsection{Destabilization of an equilibrium binary junction}

To destabilize and determine the strength of a junction, a stress $\sigma$ is applied to a stable junction. Namely, the initial configuration is comprised of five straight dislocation segments. The magnitude of the applied stress is then increased until it is found that the dislocation arms become glissile on their respective planes. The two dislocations are originally positioned $30^{\circ}$ to the junction direction $\boldsymbol{e}_{j}$. The applied stress $\boldsymbol{\sigma}$ is reverse-calculated from a superposition of the shear stresses on the glide planes that compose the junction. The shear stresses on the glide planes are assured to result in no net stress on the junction segment so that this portion 
of junction remains straight despite bowing of the dislocation arms. The superposition is given by (Wu et al., 2012, 2013):

$$
\boldsymbol{\sigma}=\sigma_{1} \mathbf{S}_{1}+\sigma_{2} \mathbf{S}_{2}
$$

in which the independent projection matrices $\boldsymbol{S}_{i}$ are employed. Here, $\sigma_{i}$ are the scalar magnitudes of stresses on each plane (i=1,2), $\boldsymbol{S}_{i}$ are obtained from the $3 \times 3$ tensor, $\boldsymbol{s}_{i}$, via:

$\boldsymbol{S}_{\mathrm{j}}: \boldsymbol{s}_{\mathrm{k}}=\boldsymbol{\delta}_{\mathrm{jk}}$

where $\boldsymbol{\delta}_{\mathrm{jk}}$ is the Kronecker delta and $\boldsymbol{s}_{\mathrm{k}}$ is given by:

$\boldsymbol{s}_{\mathrm{i}}=\left(\frac{\boldsymbol{b}_{\mathrm{i}}}{\left|\boldsymbol{b}_{\mathrm{i}}\right|} \otimes \frac{\boldsymbol{n}_{\mathrm{i}}}{\left|\boldsymbol{n}_{\mathrm{i}}\right|}+\frac{\boldsymbol{n}_{\mathrm{i}}}{\left|\boldsymbol{n}_{\mathrm{i}}\right|} \otimes \frac{\boldsymbol{b}_{\mathrm{i}}}{\left|\boldsymbol{b}_{\mathrm{i}}\right|}\right)$

which can be calculated from the Burgers vector $\boldsymbol{b}_{i}$ and the unit vector $\boldsymbol{n}_{i}(i=1,2,3)$ normal to the glide plane for each dislocation segment associated with the junction including the arms ( $i$ $=1,2)$ and the junction segment $(i=3)$.

\subsection{Material Parameters and Plane Configurations}

The junctions form at the intersection of two slip planes. The pairs of planes considered presently consist of the prismatic plane $(01 \overline{1} 0)$ and one of the remaining types of planes: basal (0001), prismatic (11̄00), Type-I (primary) pyramidal (11101), or Type-II (secondary) pyramidal ( $\overline{2} 112)$ planes. Only Burgers vectors that favor junction formation are considered by ensuring the following two criteria are met: $\boldsymbol{b}_{1}+\boldsymbol{b}_{2}=\boldsymbol{b}_{j}$ and $\left|\boldsymbol{b}_{1}\right|^{2}+\left|\boldsymbol{b}_{2}\right|^{2}>\left|\boldsymbol{b}_{j}\right|^{2}$ where $\boldsymbol{b}_{1}, \boldsymbol{b}_{2}$ and $\mathbf{b}_{j}$ are the Burgers vectors of the two intersecting dislocations and the junction, respectively. Thus, the present scope is limited to the consideration of the major basal, prismatic and pyramidal Burgers vectors corresponding to type $\langle a\rangle: \frac{1}{3}\langle 11 \overline{2} 0\rangle$, type 
$\langle c\rangle:\langle 0001\rangle$, and type $\langle a+c\rangle: \frac{1}{3}\langle 11 \overline{2} \overline{3}\rangle$. Table I summarizes the different combinations of slip systems. Pairs of plausible dislocations are considered including junctions that may result from $\langle a\rangle+\langle a\rangle,\langle a+c\rangle+\langle a\rangle$ and $\langle a+c\rangle+\langle c\rangle$ combinations. The selection of these Burgers vector pairs also applies to other binary interactions using slip systems that are not included in this work. In each case studied here, the junction is named by the intersecting planes. In the present nomenclature, the suffixes -1 and -2 are used when the same junction produced on the same intersecting edge has different pairs of interacting Burgers vectors.

\section{Table I.}

Combinations of active slip systems considered in this work.

\begin{tabular}{|c|c|c|c|c|c|}
\hline \multicolumn{3}{|c|}{ Slip plane } & \multicolumn{3}{|c|}{ Burgers vector } \\
\hline Case & 4-index & 3-index & 4-index & 3-index & type \\
\hline \multirow{3}{*}{ (1) } & (0001) & $\left(00 \frac{c}{a}\right)$ & $\frac{1}{3}[1 \overline{2} 10]$ & {$\left[\frac{1}{2} \frac{\sqrt{3}}{2} 0\right]$} & $\langle a\rangle$ \\
\hline & $(01 \overline{1} 0)$ & $(\sqrt{3} 10)$ & $\frac{1}{3}[\overline{2} 110]$ & {$\left[\frac{1}{2} \frac{-\sqrt{3}}{2} 0\right]$} & $\langle a\rangle$ \\
\hline & \multicolumn{2}{|c|}{ Basal/Prismatic Junction } & $\frac{1}{3}[\overline{1} \overline{1} 20]$ & {$[100]$} & $\langle a\rangle$ \\
\hline \multirow{3}{*}{ (2) } & $(\overline{1} 101)$ & $\left(\begin{array}{lll}0 & \frac{c}{a} & \frac{-\sqrt{3}}{2}\end{array}\right)$ & $\frac{1}{3}[\overline{1} \overline{1} 20]$ & {$[100]$} & $\langle a\rangle$ \\
\hline & $(01 \overline{1} 0)$ & $(\sqrt{3} 10)$ & $\frac{1}{3}[2 \overline{1} \overline{1} 0]$ & {$\left[\frac{-1}{2} \frac{\sqrt{3}}{2} 0\right]$} & $\langle a\rangle$ \\
\hline & Type-I Pyram & atic Junction & $\frac{1}{3}[1 \overline{2} 10]$ & {$\left[\frac{1}{2} \frac{\sqrt{3}}{2} 0\right]$} & $\langle a\rangle$ \\
\hline \multirow{4}{*}{ (3) } & $(1 \overline{1} 00)$ & $(0 \overline{1} 0)$ & $\frac{1}{3}[\overline{1} \overline{1} 23]$ & {$\left[10 \frac{c}{a}\right]$} & $\langle a+c\rangle$ \\
\hline & $(01 \overline{1} 0)$ & $(\sqrt{3} 10)$ & $\frac{1}{-}\lceil 2 \overline{1} \overline{1} \overline{3}\rceil$ & $-1 \sqrt{3}-c]$ & $\langle a+c\rangle$ \\
\hline & & & $\overline{3}^{[2110]}$ & $\overline{2} \overline{2} \bar{a}$ & \\
\hline & Prismatic & c Junction-1 & $\frac{1}{3}[1 \overline{2} 10]$ & {$\left[\frac{1}{2} \frac{\sqrt{3}}{2} 0\right]$} & $\langle a\rangle$ \\
\hline
\end{tabular}




\begin{tabular}{|c|c|c|c|c|}
\hline \multirow{3}{*}{ (4) } & $(-c \sqrt{3} c-2 a)$ & $\frac{1}{2}[2 \overline{1} \overline{1} 3]$ & {$[-1 \sqrt{3} c]$} & \multirow{3}{*}{$\begin{array}{c}\langle a+c\rangle \\
\langle a\rangle\end{array}$} \\
\hline & & 3 & {$\left[\begin{array}{lll}2 & 2 & a\end{array}\right]$} & \\
\hline & $(01 \overline{1} 0)$ & $\frac{1}{3}[\overline{2} 110]$ & {$\left[\frac{1}{2} \frac{-\sqrt{3}}{2} 0\right]$} & \\
\hline \multicolumn{2}{|r|}{ Type-II Pyramid / Prismatic Junction-1 } & [0001] & {$\left[00 \frac{c}{a}\right]$} & $\langle c\rangle$ \\
\hline \multirow{3}{*}{ (5) } & $(-c \sqrt{3} c-2 a)$ & $\frac{1}{2}[2 \overline{1} \overline{1} 3]$ & {$\left[\frac{-1}{2} \frac{\sqrt{3}}{2} \frac{c}{2}\right]$} & $\langle a+c\rangle$ \\
\hline & & & {$\left[\begin{array}{lll}2 & 2 & a\end{array}\right]$} & \\
\hline & $(\sqrt{3} 10)$ & {$[000 \overline{1}]$} & {$\left[00-\frac{c}{a}\right]$} & $\langle c\rangle$ \\
\hline \multicolumn{2}{|r|}{ Type-II Pyramidal / Prismatic Junction-2 } & $\frac{1}{3}[2 \overline{1} \overline{1} 0]$ & {$\left[\frac{-1}{2} \frac{\sqrt{3}}{2} 0\right]$} & $\langle a\rangle$ \\
\hline \multirow{3}{*}{ (6) } & $(1 \overline{1} 00)$ & $\frac{1}{3}[\overline{1} \overline{1} 2 \overline{3}]$ & {$\left[10-\frac{c}{a}\right]$} & $\langle a+c\rangle$ \\
\hline & $(\sqrt{3} 10)$ & {$[0001]$} & {$\left[00 \frac{c}{a}\right]$} & $\langle c\rangle$ \\
\hline & Prismatic / Prismatic Junction-2 & $\frac{1}{3}[\overline{1} \overline{1} 20]$ & [100] & $\langle a\rangle$ \\
\hline \multirow[t]{3}{*}{ (7) } & $\left(\begin{array}{lll}0 & \frac{c}{a} & -\frac{\sqrt{3}}{2}\end{array}\right)$ & $\frac{1}{3}[1 \overline{2} 13]$ & {$\left[\frac{1}{2} \frac{\sqrt{3}}{2} \frac{c}{a}\right]$} & $\langle a+c\rangle$ \\
\hline & $(\sqrt{3} 10)$ & {$[000 \overline{1}]$} & {$\left[00-\frac{c}{a}\right]$} & $\langle c\rangle$ \\
\hline & Type-I Pyramidal / Prismatic Junction & $\frac{1}{3}[1 \overline{2} 10]$ & {$\left[\frac{1}{2} \frac{\sqrt{3}}{2} 0\right]$} & $\langle a\rangle$ \\
\hline
\end{tabular}

The critical resolved stresses for breaking the junctions are determined by applying a stress incrementally to break an equilibrated junction. This happens when the bowing dislocations pass each other and continue gliding on their respective planes. The critical resolved stresses corresponding to this occurrence are then used to construct the junction yield surfaces. In the DD models, there are several important simulation parameters related to the calculations of nodal forces among the discretized dislocation segments. A core radius needs to be defined and compared to $r_{o}$ defined in Eq. (6) to ensure fair comparisons between the two approaches. The core energy model developed in the DD method can be improved further by incorporating more data from MD simulations such as, for instance, the core-core 
interactions, dependence of the angle between the Burgers vector and the dislocation line direction, and the effects of Burgers vector types $(\langle a\rangle,\langle c\rangle$, and $\langle c+a\rangle)$ on core energy (Iyer et al. 2014). Such a model may impact the binary interaction map and the shape of yield surface plot. This is out of the scope of this paper and is left for future work.

The cubic simulation box has a side length of $600,000 a$. All dislocations are discretized into segments ranging in length from $1,000 a$ to $3,000 a$. Seven different $h c p$ metals are studied. The material properties used in this work are listed in Table II (Newnham, 2005, Tromans, 2011).

\section{Table II}

The material properties of $h c p$ metals used in this study.

\begin{tabular}{ccccc}
\hline Material & c/a Ratio & Anisotropy Factor & Shear Modulus (GPa) & Poisson's Ratio \\
\hline $\mathrm{Cd}$ & 1.886 & 0.529 & 24.014 & 0.2799 \\
$\mathrm{Zn}$ & 1.856 & 0.610 & 39.457 & 0.2241 \\
$\mathrm{Mg}$ & 1.624 & 0.980 & 17.208 & 0.2986 \\
$\mathrm{Co}$ & 1.623 & 1.063 & 82.360 & 0.3324 \\
$\mathrm{Zr}$ & 1.593 & 0.907 & 36.026 & 0.3352 \\
$\mathrm{Ti}$ & 1.587 & 1.327 & 43.355 & 0.3431 \\
$\mathrm{Be}$ & 1.568 & 1.224 & 148.637 & 0.0657 \\
\hline
\end{tabular}

\section{Results and discussion}

The calculations and analyses are performed in two parts. First, the formation of the binary junctions are shown using $B e$ as the prototypical $h c p$ material. Secondly, junction unzipping is performed and an approximate model of the yield surface is devised.

\subsection{Maps of binary interactions and binary junctions}

The maps for binary interactions are constructed by considering the particular case of two long dislocations $\boldsymbol{A}_{1} \boldsymbol{B}_{1}$ and $\boldsymbol{A}_{2} \boldsymbol{B}_{2}$, with respective lengths $l_{1}$ and $l_{2}$. The lengths $l_{1}$ and $l_{2}$ 
Burgers vector $\boldsymbol{b}_{j}$, all of which have magnitudes of $a$. Since the Poisson's ratio of $B e$ is low ( $v$ $=0.0657$, see Table I), the junction region tends to be circular, as shown in Figs. 3(a) and 3(b). However, in Case 3, the resultant basal Burgers vector $\boldsymbol{b}_{j}$ is different and the region is no longer circular and is elongated along both axes, as indicated in Fig. 3(c) due to the larger magnitude of Burgers vectors $|a+c|$ used for both interacting dislocations. When one of the interacting dislocations possesses a Burgers vector of larger magnitude, for example a pyramidal type $\langle a+c\rangle$ or a prismatic type $\langle c\rangle$, the junction region elongates along the axis corresponding to the larger Burgers vector. In Fig. 3(d), the junction region is elongated along the $x$ axis that corresponds to the pyramidal type $\langle a+c\rangle$ dislocation used. Similarly, the junction region also elongates along the $x$ axis in Figs. 3(e) to 3(g) because a pyramidal Burgers vector is used for dislocation 1, while a prismatic Burgers vector is used for dislocation 2. Because the ratio between the pyramidal and prismatic Burgers vectors, $<a+$ $c\rangle /\langle c\rangle$, is smaller than that between the pyramidal and basal Burgers vectors, $\langle a+c\rangle /$ $\langle a\rangle$, the aspect ratio of the dark region in Figs. 3(e)-3(g) is smaller than in Fig. 3(d).

As apparent in Fig. 3, the junction regions appear to dominate the map in several cases (Cases 3, 5-7). By varying the orientations $\phi_{i o}(i=1,2)$ of intersecting dislocations, junction formation is favored when the combined arm line tension exceeds the junction line tension along the junction direction $\boldsymbol{e}_{j}$ at the triple node, namely $F_{e q}>0$. Therefore, the formation can be the results of both attractive and repulsive interactions, $F_{12}\left(\phi_{1 o}, \phi_{2 o}\right)$. The preponderance of dark regions in Case 3 and Cases 5-7 in Fig. 3 also indicates that type $\langle c\rangle$ dislocations prefer to form junctions when a type $\langle a\rangle$ junction is accessible. Our results suggest that this trend appears to be independent of slip system. 

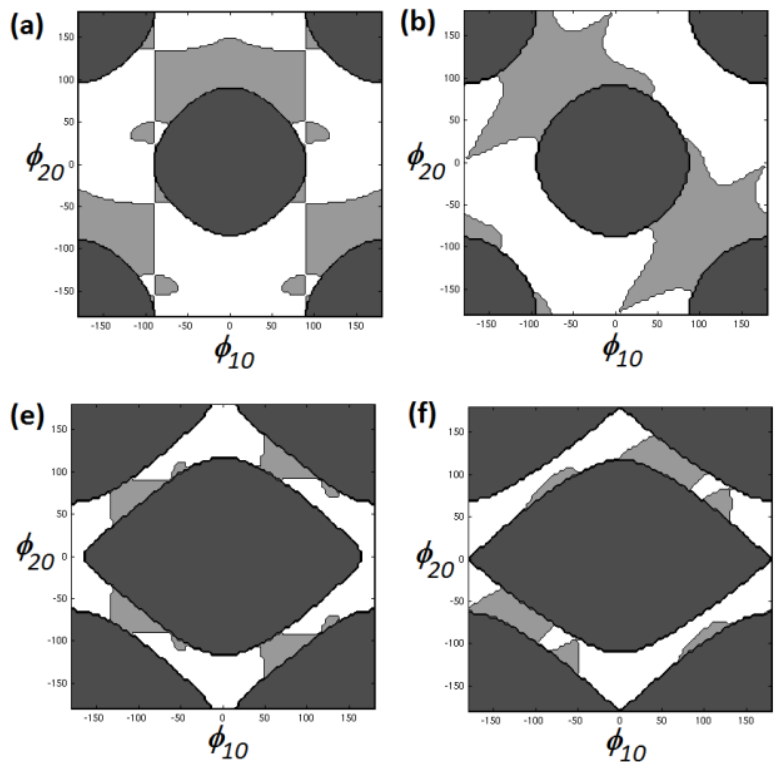
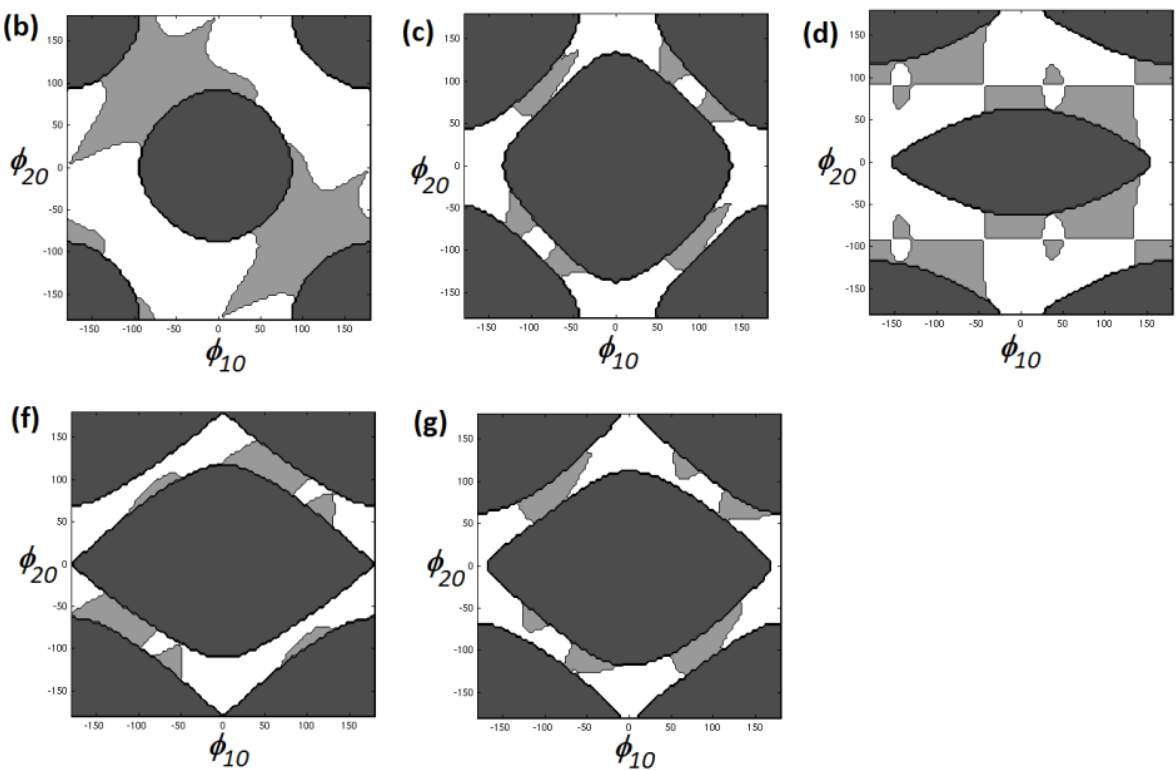

Fig. 3. The maps of binary dislocation interactions for $B e$ via the analytical approach of line tension approximation: (a) Case 1, (b) Case 2, (c) Case 3, (d) Case 4, (e) Case 5, (f) Case 6 and (g) Case 7. Cases are defined in Table II. Dark, light and unshaded regions signify junction formation, cross-states and no junction states, respectively.

From the maps of binary interactions in Fig. 3, sensitivity of the shapes and sizes of the light and unshaded regions to the glide planes are observed independent of the Burgers vectors. For example, pairs of two basal Burgers vectors of type $\langle a\rangle$ are used for Cases 1,2 , and 3 in Figs. 3(a) and 3(b) and pairs of pyramidal type $\langle a+c\rangle$ and prismatic type $\langle c\rangle$ Burgers vectors are used for Cases 5, 6, and 7 in Fig. 3 (e) to (g). They show the same shape of junction regions but different cross-state regions (light regions).

The binary interactions predicted by the DD simulations and line-tension model are largely in agreement. Using Case 1, Fig. 4 depicts a superposition of the analytical map of Fig. 3(a) and the numerical junction maps computed via DD shown as symbols using a $13 \times 13$ grid of points each point corresponding to a pair of angles. In this figure, the filled black circles and crosses represent $G=1$ and $G=0$, representing junctions and cross-states, respectively. 
This agreement between the analytical and DD-simulation approaches suggest that it is possible to predict junction formation from binary dislocation interactions using the line tension model alone. The interaction map consistently shows junction-favored regions at the center and four corners regardless of the crystal type, at least among hcp and cubic crystals. For example, Capolungo (2011) reported binary interaction maps for $\mathrm{Mg}$ that are in agreement with Fig. 3(a). The symmetry in Figs. 3(a) to 3(c) is also consistent with previous findings in $f c c$ metals (Wickham et al., 1999) using Burgers vectors of the same type (both type $\langle a\rangle$ for Figs. 3(a) and 3(b) and type $\langle a+c\rangle$ for Fig. 3(c)).

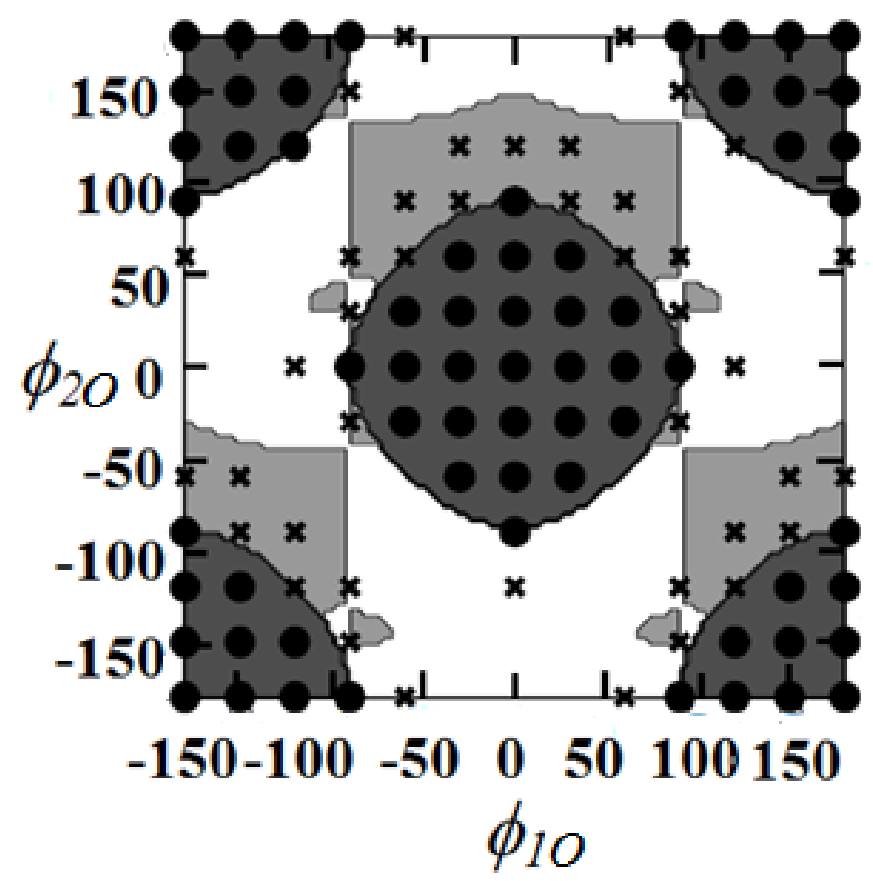

Fig. 4. The maps of binary dislocation interactions for $B e$ between the DD and line tension model for Case 1. Continuous dark, light, and unshaded regions correspond to junction formation, cross-states and repulsive states. Filled black circles and crosses represent $G=1$ and $G=0$, respectively, via the DD method using a total 13 increments each for the angle pairs $\left(\phi_{1}, \phi_{2}\right)$. 


\subsection{Junction unzipping and yield surfaces}

The strength of the junctions is next evaluated by producing yield surface plots as a function of the resolved stresses on each of the slip planes required to reverse the formation mechanism of the junction. Stable junctions can be produced for any arbitrary $h c p$ material using the procedures outlined above. Then, upon the application of an externally imposed stress field, the junction can be made to "unzip" eventually returning to its two-dislocation form. As a rule of thumb, the critical resolved stresses to break a junction tend to increase with the material's elastic shear modulus. Therefore, a normalization factor is introduced to enable comparisons among different materials:

$$
\sigma_{i, c}=\sigma_{i, c}^{\prime}\left[\frac{\mu a}{l_{i o}(1-v)} \ln \left(\frac{R}{r_{o}}\right)\right]
$$

where $l_{i_{o}}$ is the initial length of dislocation $i$ (for $i=1,2$ ), $a$ is the lattice spacing, and $\sigma_{i, c}$ and $\sigma_{i, c}^{\prime}$ are the resolved stresses before and after normalization, respectively. The term " $a$ " is in the numerator because $l_{i o}(=40,000 a)$ is in units of $a$. Thus, the normalized critical stresses $\sigma_{i, c}^{\prime}$ are dimensionless. The critical stresses $\left(\sigma_{1, c}, \sigma_{2 . c}\right)$ are the minimum resolved stress needed to completely unzip a binary junction. The normalized critical stresses ( $\sigma_{1, c}^{\prime}$, $\left.\sigma_{2, c}^{\prime}\right)$ are used to construct the yield surfaces in the space of normalized resolved stresses on both slip planes.

The yield surface plot depicts stress needed to de-stabilize a junction. Therefore, the size and shape of the yield surface are quantitative indicators for possible junction reactions in stressed crystals. The destabilization of the junction is modeled using a balance of forces 
at a junction triple node. The critical applied stress needed to break the junction exceeds the critical dislocation bow out stress. It is therefore likely that the bow-out may have an effect on magnitude of the critical stress required to destabilize the junction. $h c p$ metals with a wide range of elastic properties are studied (see Table I). The size of a junction yield surface is found to be dependent on the Poisson's ratio. This can be attributed to the linear relation between the critical stresses $\sigma_{i, c}$ and the shear modulus $\mu$ and involvement of Poisson's ratio in the normalization factor, as indicated in Eq. (36). Among all the $h c p$ metals and slip systems studied in this work, the resultant yield surfaces are remarkably similar. The effect of differing elastic properties appear in Fig. 5 for $B e$ and $M g$. The Poisson's ratio, $v$, clearly affects the size of the yield surface. The yield surfaces for $M g$ are categorically smaller than for $B e$. In addition, the yield surface plots in all cases show strong rotational symmetries. The symmetry of yield surface plots was anticipated given the symmetric configurations for the equilibrium junction used at the beginning of the unzipping process (Wu et al., 2013) with the isotropic linear elasticity assumption. The symmetry of yield surfaces has also been reported for cubic systems (Shenoy et al., 2000; Dupuy and Fivel, 2002). 

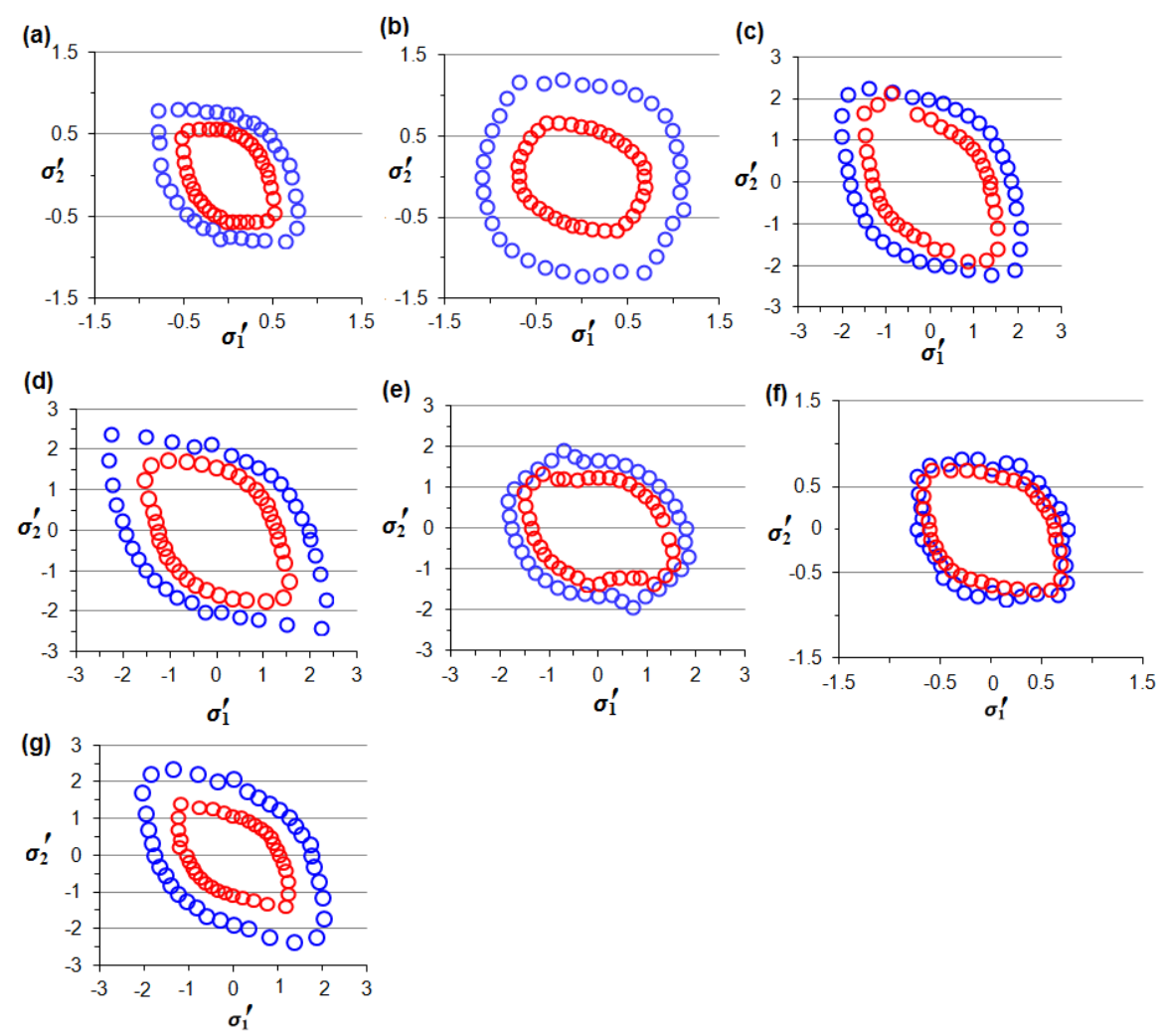

Fig. 5. The yield surfaces of all different binary junctions calculated by DD simulations with full elasticity for Be (blue) and $M g$ (red). (a) Case 1; (b) Case 2 ; (c) Case 3 ; (d) Case 4; (e) Case 5; (f) Case 6; (g) Case 7. The $\sigma_{1}$ ' and $\sigma_{2}$ ' are the normalized resolved applied stresses on the first and second slip planes, respectively.

Comparing the yield surfaces using the normalized stresses exposes notable similarities. In all of the cases, the general shape and tilting angle are consistent across different materials that have a similar junction configuration. This will be discussed further along with the analytical results in Sections 3.3 and 3.4. Figure 6 depicts one of these comparisons for the yield surfaces for a junction formed from dislocations where both are type $\langle a\rangle$, namely the $(0001)[1 \overline{2} 10] /(01 \overline{1} 0)[\overline{2} 110]$ junction in Case 1 . The figure shows extraordinary uniformity of shapes among the seven different $h c p$ metals studied. In comparison to Fig. 5 (a), Fig. 6 also emphasizes the significantly larger envelope for Be due 
to its considerably smaller Poisson's ratio (0.066) than those of other $h c p$ metals studied having comparable Poisson's ratios varying from 0.224 to 0.343 .

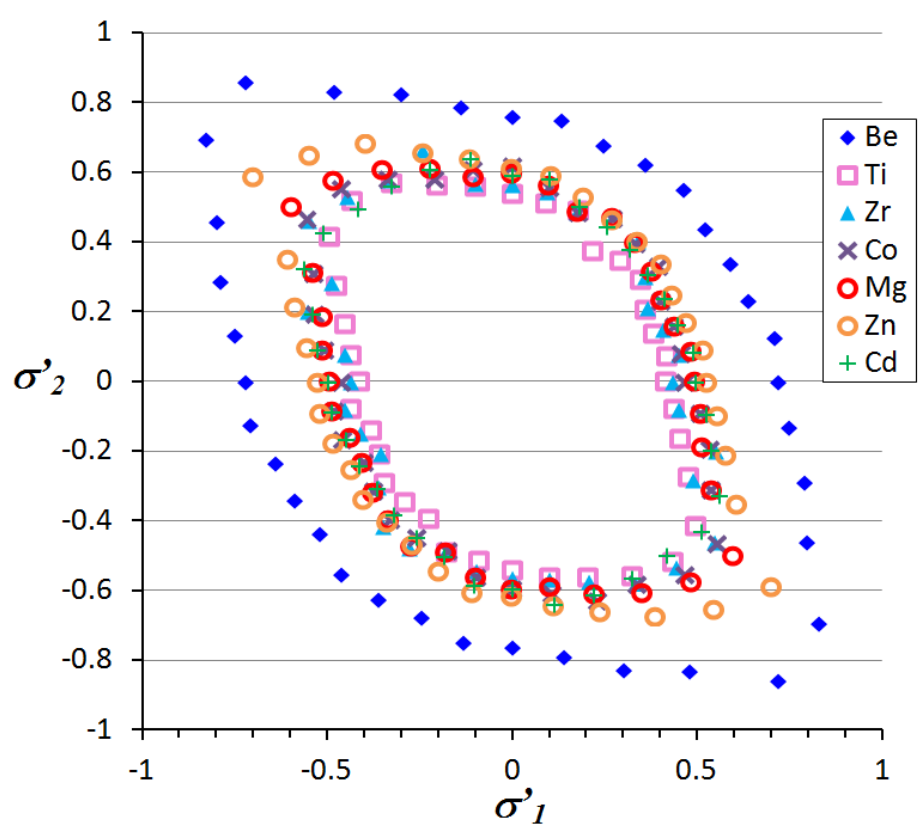

Fig. 6. The superposition of yield surfaces for the (0001)[12 10$] /$ (0110) $[\overline{2} 110]$ junction using both basal $\langle a\rangle$-type Burgers vectors.

The binary dislocation junction forms when it is energetically favorable to do so. A larger reduction in the total energy generally results in a junction that requires a larger stress to "unzip". This is due to the proportional relation between the dislocation energy and the square of Burgers vector magnitude, as indicated in Eq. (6). Because of the differences in the magnitude among basal, prismatic, and pyramidal types of Burgers vectors for $h c p$ crystals, junction strength can be approximated by comparing the differences in the magnitude between the Burgers vectors used for dislocation arms and the Burgers vector of the junction. For $f c c$ and $b c c$ crystals, however, the total energy reduction due to junction formation is independent of interacting slip planes and Burgers vectors because all Burgers vectors have the same magnitude. The extent of differences in the 
Burgers vector magnitude depends on the $c l a$ ratio for $h c p$ metals. Therefore, the junction strength depends on the $c / a$ ratio as well. The magnitude of the pyramidal type $\langle a+c\rangle$ Burgers vector is approximately two times larger than the basal type $\langle a\rangle$ Burgers vector and approximately $1.1 \sim 1.2$ greater than the prismatic type $\langle c\rangle$ Burgers vector. Therefore, among the cases considered presently, a junction with a basal Burgers vector produced from two pyramidal Burgers vectors is the strongest since it corresponds to the largest change in Burgers vector magnitudes from the formation. The trend follows similarly for a junction having a basal Burgers vector formed by a pyramidal and a prismatic Burgers vectors. Among the cases in Table I, junctions with a prismatic Burgers vector formed by one pyramidal and one basal Burgers vectors are weakest because the total energy reduction is comparatively much smaller. Junctions with a type $\langle a\rangle$ Burgers vector produced from two dislocations both with basal Burgers vectors consistently reduces the total energy by $50 \%$ for all of the materials presently considered. This is similar to what happens in cubic crystals where the junctions are formed by and result in dislocations that have the same Burgers vectors. However, it is different for $f c c$ than $b c c$ crystals in terms of energy reduction from binary reactions of like Burgers vectors. The quantitative effects on the total energy reduction by varying the Burgers vector type and the material $c / a$ ratio are summarized in Table III. As shown in the table, the larger the cla ratio, the greater the differences in the total energy reduction and, therefore, the larger the junction strength. For example, $C d$ and $Z n$ possess the largest $c / a$ ratios (1.8862 and 1.8563, respectively). Almost 90\% of the total energy can be reduced by forming a binary junction in Case 3 and only $\sim 36 \%$ can be reduced if the binary junction is formed using the interacting slip systems in Case 4. For other metals, the total energy reduction from pyramidal/basal 
interactions is comparatively higher ( $44 \%)$ due to their comparable $c / a$ ratios around 1.630.

Table III.

The total energy reduction in percent via the formation of binary dislocation junctions using

\begin{tabular}{ccccc}
\hline Material & $\begin{array}{c}\langle a+c\rangle /\langle a+c\rangle \\
\rightarrow\langle a\rangle\end{array}$ & $\begin{array}{c}\langle a+c\rangle /\langle c\rangle \\
\rightarrow\langle a\rangle\end{array}$ & $\begin{array}{c}\langle a+c\rangle /\langle a\rangle \rightarrow\langle c\rangle \\
\text { Case } 4\end{array}$ & $\begin{array}{c}\langle a\rangle /\langle a\rangle \rightarrow\langle a\rangle \\
\text { Cases 1,2 }\end{array}$ \\
\hline $\mathrm{Cd}$ & 89.03 & 87.68 & 35.99 & 50 \\
$\mathrm{Zn}$ & 88.75 & 87.33 & 36.73 & 50 \\
$\mathrm{Mg}$ & 86.25 & 84.06 & 43.14 & 50 \\
$\mathrm{Co}$ & 86.24 & 84.04 & 43.16 & 50 \\
$\mathrm{Zr}$ & 85.86 & 83.54 & 44.08 & 50 \\
$\mathrm{Ti}$ & 85.79 & 83.44 & 44.25 & 50 \\
$\mathrm{Be}$ & 85.54 & 83.10 & 44.85 & 50 \\
\hline
\end{tabular}

different interacting and resultant Burgers vectors. The numbers are approximated based on the proportional relation between the energy of a dislocation and the magnitude of the Burgers vector.

\subsection{Effects of dislocation interactions and universal comparison ratio $\eta$}

Whereas the line-tension model neglects the energy contribution from dislocation interactions, the present DD simulations account for them based on the Kroupa formula as shown in Eq. (1) (Arsenlis et al., 2007). To investigate the effects of dislocation interactions on the strength of junctions, DD simulations are performed for $B e$ and $M g$ using conditions identical to those employed in the line-tension model. The two hcp metals were chosen so as to examine the role of elastic properties since they have drastically different Poisson's ratios (0.0657 and 0.2986, for Be and $\mathrm{Mg}$, respectively). In each of the panels in Figs 7 and 8, three sets of results are shown. The blue line indicates the line tension model result with a small dislocation bow out under the applied stress field. The open blue symbols are from the DD simulations with the line tension. The solid red symbols are with the interactions 
included. In the absence of the dislocation segment-segment interactions, DD simulations generally are in good agreement with the line tension model approximation.

The noticeable differences may be attributed to the large distortions of the arms due to Frank-Read bow-out that is accounted for in the DD simulation results but are assumed small in the line-tension model. The bow-out occurs when the system is subjected to the externally imposed stress field. Thus it may be possible to improve the agreement by taking into account the bowing arm and incorporating large bow-out angle at the cost of making the expressions lengthier. Figures 7 and 8 prompt two noteworthy observations. First, despite differences in the yield surfaces that are obviously due to the differences in the different slip systems used for intersecting dislocations (as shown in Table I), there is remarkable qualitative consistency in the shapes between the DD simulations and the line tension model. The similarity holds regardless of the case, the material, and the inclusion of segment-segment interactions. The results verify the agreement between the line tension force in the model and the core force developed by the non-singular theory for a dislocation loop and used in DD simulations (Cai et al., 2006). The results also indicate that full elasticity may not be necessary to predict the strength of a binary junction via DD simulations because dislocation line tension dominates dislocation interactions for simple dislocation configurations such as binary junctions. However, such elastic interactions among each possible pair of dislocation segments would become increasingly significant and contribute to the overall plasticity with the growth of dislocation population. The line tension model assumes a Burgers vector corresponding to the shortest lattice translation on the most densely-packed glide plane in an ideally-packed isotropic medium without taking into account the dislocation bow out. For Be, a complete match of the yield surface plots 
Fig. 7. Yield surfaces calculated with different approaches for Be. The solid red symbols are yield surface points calculated using DD simulations with full elasticity. The open blue symbols and continuous lines are those determined using the line tension model from the simulation and the analytical approach, respectively. (a) Case 1; (b) Case 2 ; (c) Case 3 ; 
(d) Case 4; (e) Case 5; (f) Case 6; (g) Case 7. The $\sigma_{1}$ and $\sigma_{2}$ are the resolved applied stresses in the unit of $\mathrm{MPa}$ on the first and second slip planes, respectively.
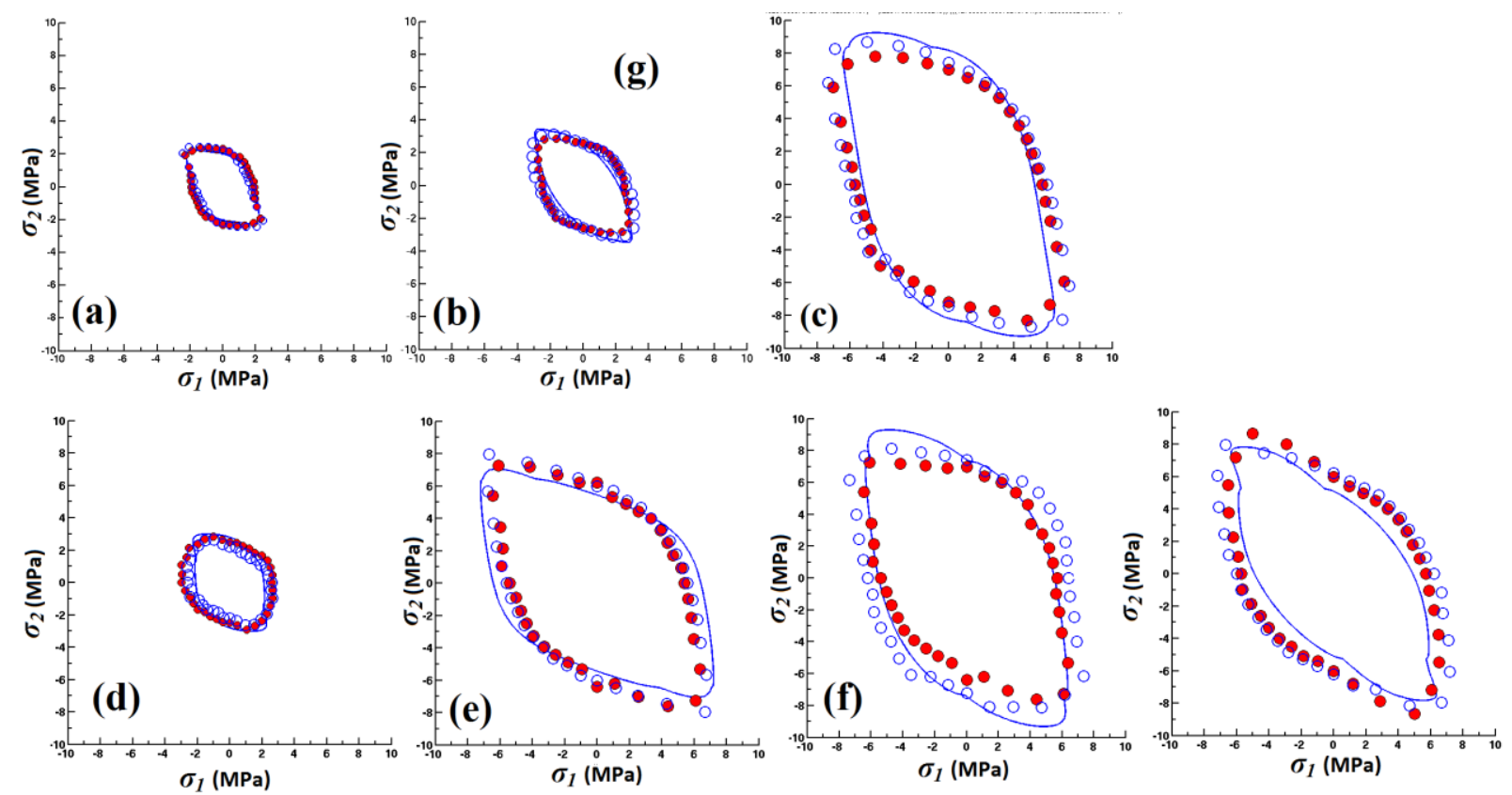

Fig. 8. Yield surfaces calculated with different approaches for $\mathrm{Mg}$. The solid red symbols are yield surface points calculated using DD simulations with full elasticity. The open blue symbols and continuous lines are those determined using the line tension model from the simulation and the analytical approach, respectively. (a) Case 1; (b) Case 2 ; (c) Case 3 ; (d) Case 4; (e) Case 5; (f) Case 6; (g) Case 7. The $\sigma_{1}$ and $\sigma_{2}$ are the resolved applied stresses in the unit of MPa on the first and second slip planes, respectively.

\subsection{A regressive model for junction yield surfaces}

Based on the observations described in Section 3.3, we find that the analytical line tension balance approach can reasonably approximate the yield surface points obtained from DD simulations. A single comparison ratio, $\eta$, is used to quantitatively compare the data between the model and DD simulations among all tested hop metals. The ratio is calculated by systematically taking the individual ratios of the net stress magnitudes, $\sqrt{\sigma_{1, c}^{2}+\sigma_{2, c}^{2}}$, of the critical stress pairs $\left(\sigma_{1, c}, \sigma_{2, c}\right)$ obtained from the DD simulation to the analytical approach 
and then computing the mean value over all possible stress pairs for each case. The standard deviations are indicated by the error bars. The results indicate that $\eta \approx 1$ for any dislocation pair and any of the hep materials presently considered, as shown in Fig. 9. The observed ratio also demonstrates the independence of elastic effects on the strength of binary junctions given no interferences from any other dislocations. There may be an intrinsic strength that is overcome by the combination of the applied stress and the local stress field created by the other segments. The agreement of computed $\eta$ values also echoes the yield surface plots presented in Fig. 7 for $B e$ and Fig. 8 for $M g$ with the extension to include other $h c p$ metals of varying extents of anisotropy and ideal packing ( $c / a$ ratio).

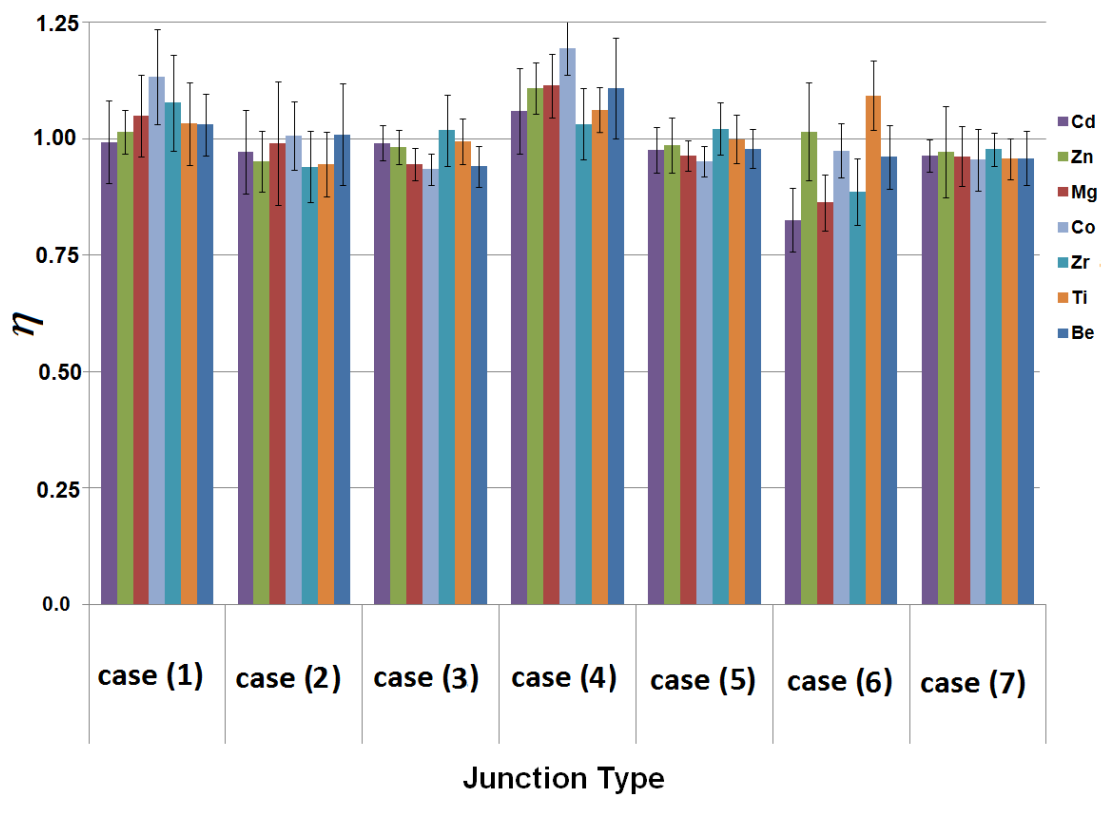

Fig. 9. Comparison factors relating points on the line tension model yield surface, which neglects segment interactions, to the DD simulations where the segment interactions are included. Bar height indicates mean over all points in the computed yield surface. Error bars signify deviations.

The junction yield surface is a locus of points in the stress space that indicates the pair of stresses on the respective slip planes of the two dislocations required to de-stabilize the junction. The yield surfaces examined in this work all display an oblique appearance 
along the negative diagonal to varying extents of elongation. The junction becomes unstable when the forces on the multi-node no longer sum to zero or, namely, when the stress state is not on the interior of the enclosed locus. The continuous blue curves in Figs. 7 and 8 represent the yield surface and come from the solutions of the critical stress pair $\left(\sigma_{1, \mathrm{c}} \sigma_{2, c}\right)$ to two polynomial equations, one of which was shown in Eq. (19). They are evolved from the solution of two third-order polynomials.

Based on the remarkably consistent shape of the yield surface, we introduce an approximate expression to generate a yield surface based on Eq. (19) which we treat as a polynomial having the same major and minor axes of a reclined ellipse, Eq. (24). As shown in Sec. 2.3.2, the titling angle $\beta$, can be first estimated using Eq. (31). Then, $\beta$ can be substituted into Eqs. (27) and (28) along with given parameters including Burgers vectors, $b_{i}$, and initial dislocation characters, $\theta_{i}$ to obtain the major and minor axes, $p$ and $q$ that are essentially the longest and shortest lines drawn from one side of the yield surface to the opposite through the center point $(0,0)$. As an example, the aspect ratio and tilting angle for each junction configuration for $B e$ and $M g$ are shown in Table IV.

Table IV. Aspect ratios and tilting angles of yield surface plots for junctions examined for $B e$ and $M g$.

\begin{tabular}{ccccc}
\hline Material & \multicolumn{2}{c}{$B e$} & \multicolumn{2}{c}{$M g$} \\
\hline $\begin{array}{c}\text { Junction } \\
\text { configuration }\end{array}$ & $\begin{array}{c}\text { Tilting angle } \beta \\
\text { in degrees }\end{array}$ & Aspect ratio & $\begin{array}{c}\text { Tilting angle } \\
\beta \text { in degrees }\end{array}$ & Aspect ratio \\
\hline Case 1 & 44.1 & 1.18 & 44.8 & 1.23 \\
Case 2 & 40.5 & 1.15 & 39.5 & 1.55 \\
Case 3 & 44.6 & 1.55 & 42.9 & 1.45 \\
Case 4 & 35.3 & 1.89 & 26.3 & 1.05 \\
Case 5 & 43.2 & 1.36 & 37.3 & 1.37 \\
Case 6 & 44.6 & 1.37 & 43.5 & 1.08 \\
Case 7 & 44.0 & 1.35 & 35.5 & 1.49 \\
\hline
\end{tabular}


mathematical expression for the coefficient of each term makes such a direct use unfavorable even though it mathematically describes a yield surface as function of stresses. On the other hand, it is more practical to quantitatively correlate geometric factors such as aspect ratio and tilting angle to identifiable parameters such as Burgers vectors and elastic constants.

\section{Conclusion}

We have comprehensively investigated binary dislocation interactions in hcp metals using DD models and an analytical approach based on the modified orientation-dependent line tension model. The analytically-computed junction maps show the Burgers vectors of the dislocations that comprise the junction strongly influence the symmetry and size of junctionfavoring regions in the maps. When the intersecting dislocations possess Burgers vectors of the same type, the junction-favoring region are in the center and in the corners of the map and are arranged in a two-fold symmetric form. The junction regions elongate along the axis corresponding to the larger magnitude of Burgers vector. The strengths of seven different junction configurations have also been studied across seven different $h c p$ metals for a total of 49 different samples. Regardless of the changes in the interacting slip systems as well as the material elastic constants, the binary junctions we studied have extraordinarily similar yield surface plots in terms of shape and orientation. Because dislocation line tension dominates in the total elastic energy in binary dislocation junctions, DD simulations with and without elastic dislocation interactions predict similar junction strength with nearly identical sizes of junction yield surfaces for all cases we examined. This was further confirmed by the ratio of $\eta \sim 1$ over all possible stress pairs for any dislocation pairs and any of the hcp materials presently considered. This $\eta$ ratio also demonstrates the independence of dislocation 
interactions on the intrinsic junction strengths. This holds even with changes in the local stress due to dislocation segment evolution during unzipping under the externally applied stress field. In general, the line-tension model is able to accurately estimate the strength of a binary junction. In the comparison between $B e$ and $M g$, the two $h c p$ metals having different anisotropy and $c l a$ ratios, for $B e$ the junction strength is roughly $30 \%$ smaller at certain stress conditions when one or both of the interacting Burgers vectors are not type $\langle a\rangle$. On the other hand, for $M g$ all junction yield surfaces agree well at any stress condition. An empirical polynomial equation was developed from the line tension model to compare with the DD simulation results. The equation was treated as a degenerated super ellipse equation to quantify the aspect ratio and tilting angle of junction yield surface plots.

\section{Acknowledgement}

The corresponding author Chi-Chin Wu would like to acknowledge the support from the Secure Mission Solutions, Inc. (N65235-06-D8847) and Oak Ridge Institute for Science and Education Program in Maryland (ORISE-1120-1120-99). The simulations in this article by authors $\mathrm{Wu}$ and Chung were performed using the computing resources provided by the US Army Research Laboratory (ARL) High Performance Supercomputing Resource Center (DSRC). The efforts of binary interaction maps by authors Aubry and Arsenlis were performed under the auspices of the U.S. Department of Energy by Lawrence Livermore National Laboratory under contract No. DE-AC52-07NA27344. Author Chung would also like to acknowledge partial support from the Computational Sciences Division of ARL, the Army Research Office (W911NF1410330) and the Department of Mechanical Engineering, University of Maryland at College Park. 


\section{References}

Akarapu, S., Zbib, H.M., Bahr, D.F., 2010. Analysis of heterogeneous deformation and dislocation dynamics in single crystal micropillars under compression. Int. J. Plasticity. 26, 239-257.

Akhtar, A., 1975. Prismatic slip in zirconium single crystals at elevated temperatures. Metall. Trans. A 6A, 1217-1222.

Ando, S., Gotoh, T., Tonda, H., 2002. Molecular dynamics simulation of $\langle c+a\rangle$ dislocation core structure in hexagonal-close-packed metals, Metall. Mater. Trans. A 33A, 823829.

Arsenlis, A., Cai, W., Tang, M., Rhee, M., Oppelstrup, T., Hommes, G., Pierce, T.G., Bulatov, V.V., 2007. Enabling strain hardening simulations with dislocation dynamics. Modelling Simul. Mater. Sci. Eng. 15, 553-595.

Aubry, S., Rhee, M., Hommes, G., Bulatov, V.V., Arsenlis, A., Dislocation dynamics in hexagonal closed-packed crystals, submitted to J. Mech. Phys. Solids.

Benzerga, A.A., Bréchet, Y., Needleman, A., Van der Giessen, E., 2004. Incorporating threedimensional mechanisms into two-dimensional dislocation dynamics. Modelling Simul. Mater. Sci. Eng. 12, 159-196.

Bertin, N., Capolungo, L., Beyerlein, I.J., 2013. Hybrid dislocation dynamics based strain hardening constitutive model. Int. J. Plasticity 49, 119-144.

Bertin, N., Tomé, C.N., Beyerlein, I.J., Barnett, M.R., Capolungo, L., 2014. On the strength of dislocation interactions and their effect on latent hardening in pure magnesium. Int. J. Plasticity 62, 72-92. 
Bulatov, V., Abraham, F.F., Kubin, L., Devincre, B., Yip, S., 1998. Connecting atomistic and mesoscale simulations of crystal plasticity. Nature 391, 669-672.

Bulatov, V.V., Hsiung, L.L., Tang, M., Arsenlis, A., Bartelt, M.C., Cai, W., Florando, J.N., Hiratani, M., Rhee, M., Hommes, G., Pierce, T.G., Díaz de la Rubia, T., 2006. Dislocation multi-junctions and strain hardening. Nature 440, 1174-1178.

Cai, W., Arsenlis, A., Weinberger, C.R., Bulatov, V.V., 2006. A Non-singular continuum theory of dislocations. J. Mech. Phys. Solids 54, 561-587.

Capolungo, L., 2011. Dislocation junction formation and strength in magnesium. Acta Materialia 59, 2909-2917.

Chaari, N., Clouet, E., Rodney, D., 2014. First-principles study of secondary slip in zirconium. Phys. Rev. Lett. 112, 075504 (5pp).

Damiano, V.V., 1963. The double tetrahedron - a method of notation for Cph structures. Trans. Metall. Soc. AIME 227, 788-789.

Devincre, B., Hoc, T., Kubin, L., 2008. Dislocation mean free paths and strain hardening of crystals. Science 320, 1745-1748.

Devincre, B., Kubin, L.P., 1994. Simulations of forest interactions and strain hardening in FCC crystals. Modelling Simul. Mater. Sci. Eng. 2, 559-570.

de Wit, G. and Koehler, J.S., 1959. Interaction of dislocations with an applied stress in anisotropic crystals. Phys. Rev. 116, 1113-1120.

Dupuy, L., Fivel, M.C., 2002. A study of dislocation junctions in FCC metals by an orientation dependent line tension model. Acta Materialia 50, 4873-4885. 
Gao Y., Zhuang, Z., Liu, Z.L., You, X.C., Zhao, X.C., Zhang, Z.H. 2011. Investigations of pipe-diffusion-based dislocation climb by discrete dislocation dynamics. Int. J. Plasticity 27, 1055-1071.

Grinberg, B.A., Ivanov, M.A., Antonova, O.V., Vlasova, A.M., Kruglikov, N.A., Plotnikov, A.V., 2012. Detection of $(c+a)$ type dislocation self-blocking in magnesium, Russ. Phys. J. 54, 906-913.

Groh, S., Martin, E.B., Horstemeyer, M.F., Bammann, D.J., 2009. Dislocation motion in magnesium: a study by molecular statistics and molecular dynamics. Modelling Simul. Mater. Sci. Eng. 17, 075009 (15pp).

Groh, S., Martin, E.B., Horstemeyer, Zbib, H.M., 2009. Multiscale Modeling of the plasticity in an aluminum single crystal. Int. J. Plasticity 25, 1456-1473.

Hirth, J.P., 1961. On dislocation interactions in the fcc lattice. J. Appl. Phys. 32, 700-706.

Hirth, J.P., Lothe, J., 1982. Theory of Dislocations, Chaps. 3, 6, 20, 22, second ed. Krieger Publishing Company.

Hull, D. and Bacon, D.J., 1984. Introduction to Dislocations, Chap. 4, Third ed. Butterworth Heinemann.

Huang, M., Zhao, L., Tong, J., 2012. Discrete dislocation dynamics modeling of mechanical deformation of nickel-based single crystal superalloys. Int. J. Plasticity 28, 141-158.

Iyer, M., Radhakrishnan, B., Gavini, V., 2014. Electronic-structure study of an edge dislocation in aluminum and the role of macroscopic deformations on its energetic. J. Mech. Phys. Solids 76, 260-275.

Kalman, D., 2008. The Mmost marvelous theorem in mathematics, The Journal of Online Mathematics and its Applications. 
Kroupa, F., 1961. The force between non-parallel dislocations. Czech J. Phys. B 11, 847-848.

Lee, S.W., Aubry, S., Nix, W.D., Cai, W., 2011. Dislocation junctions and jogs in a freestanding FCC thin film. Modelling Simul. Mater. Sci. Eng. 19, 025002 (14pp).

Liu, Z.L., Liu, X.M., Zhuang, Z., You, X.C., 2009. A multi-scale computational model of crystal plasticity at submicron-to-nanometer scales. Int. J. Plasticity 25, 1436-1455.

Madec, R., Devincre, B., Kubin, L.P., 2002. On the nature of attractive dislocation crossed states. Comput. Mater. Sci. 23, 219-224.

Monnet, G., Devincre, B., Kubin, L.P., 2004. Dislocation study of prismatic slip systems and their interactions in hexagonal close packed metals: application to zirconium. Acta Materialia 52, 4317-4328.

Motz, C., Weygand, D., Senger, J., Gumbsch, P., 2009. Initial dislocation structures in 3-D discrete dislocation dynamics and their influence on microscale plasticity. Acta Materialia 57, 1744-1754.

Newnham, R.E., 2005. Properties of Materials: Anisotropy, Symmetry, Structure, Chap. 13, Oxford University Press.

Pontes, J., Walgraef, D., Aifantis, E.C., 2006. On dislocation patterning multiple slip effects in the rate equation approach. Int. J. Plasticity 22, 1486-1505.

Rao, S.I., Dimiduk, D.M., Parthasarathy, T.A., El-Awady, J., Woodward, C., Uchic, M.D., 2011. Calculations of intersection cross-slip activation energies in fcc metals using nudged elastic band method. Acta Mater. 59, 7135-7144.

Rodney, D., Phillips, R., 1999. Structure and strength of dislocation junctions: an atomic level analysis. Phys. Rev. Lett. 82, 1704-1707. 
Schoeck, G, Frydman, R., 1972. The contribution of the dislocation forest to the flow stress. Phys. Stat. Sol. (b) 53, 661-673.

Shenoy, V.B., Kukta, R.V., Phillips, R., 2000. Mesoscopic analysis of structure and strength of dislocation junctions in fcc metals. Phys. Rev. Lett. 84, 1491-1494.

Tromans,D., 2011. Elastic anisotropy of hcp metal crystals and polycrystals. IJRRAS, 6, 462483.

Wang, H., Wu, P.D., Wang, J., Tomé, C.N., 2013. A crystal plasticity model for hexagonal close-packed (HCP) crystals including twinning and de-twinning mechanisms. Int. J. Plasticity 49, 36-52.

Wickham, L.K., Schwarz, K.W., Stölken, J.S., 1999. Rules for forest interactions between dislocations. Phys. Rev. Lett. 83, 4574-4577.

Wu, C.-C., Aubry, S., Chung, P.W., Arsenlis, A., 2012. Dislocation dynamics simulations of junctions in hexagonal close-packed crystals. MRS Proceedings 1424, mrsf11-1424ss07-37 doi:10.1557/opl.2012.234.

Wu, C.-C., Chung, P.W., Aubry, S., Munday, L.B., Arsenlis, A., 2013. The strength of binary junctions in hexagonal close-packed crystals. Acta Materialia 61, 3422-3431.

Yoo, M.H., Agnew, S.R., Morris, J.R., Ho, K.M., 2001. Non-basal slip systems in HCP metals and alloys: source mechanisms. Mater. Sci. Eng. A 319-321, 87-92.

Zbib, H.M., Díaz de la Rubia, T., Rhee, M., Hirth, J.P., 2000. 3D dislocation dynamics: stress-strain behavior and hardening mechanisms in fcc and bcc metals. J. Nuclear Mater. 276, 154-165.

Zhou, C., Beyerlein, I.J., LeSar, R., 2011. Plastic deformation mechanisms of fcc single crystals at small scales. Acta Materialia 59, 7673-7682. 
Zhou, C., LeSar, R., 2012. Dislocation dynamics simulations of plasticity in polycrystalline thin films. Int. J. Plasticity 30-31, 185-201. 